\title{
Fuchsian Triangle Groups and Grothendieck Dessins. Variations on a Theme of Belyi
}

\author{
Paula Beazley Cohen ${ }^{1}$, Claude Itzykson ${ }^{2}$, Jürgen Wolfart ${ }^{3}$ \\ 1 URA 747 CNRS, Collège de France, 3 rue d'Ulm, F-75231 Paris Cedex 05, France \\ 2 Service de Physique Théorique, Centre d'études de Saclay, F-91191 Gif-sur-Yvette Cedex, France \\ 3 Math. Seminar der Univ., Robert-Mayer-Strasse 10, D-60054 Frankfurt a. M., Germany
}

Received: 13 May 1993/in revised form: 22 September 1993

\begin{abstract}
According to a theorem of Belyi, a smooth projective algebraic curve is defined over a number field if and only if there exists a non-constant element of its function field ramified only over 0,1 and $\infty$. The existence of such a Belyi function is equivalent to that of a representation of the curve as a possibly compactified quotient space of the Poincaré upper half plane by a subgroup of finite index in a Fuchsian triangle group. On the other hand, Fuchsian triangle groups arise in many contexts, such as in the theory of hypergeometric functions and certain triangular billiard problems, which would appear at first sight to have no relation to the Galois problems that motivated the above discovery of Belyi. In this note we review several results related to Belyi's theorem and we develop certain aspects giving examples. For preliminary accounts, see the preprint [Wo1], the conference proceedings article [BauItz] and the "Comptes Rendus" note [CoWo2].
\end{abstract}

\section{Introduction}

While several years ago the moduli space of compact Riemann surfaces seemed to be as remote a subject of consideration in theoretical physics as possible, it plays nowadays a considerable role from various points of view. To name some: string field theory, conformal statistical physics, topological field theories, two dimensional quantum gravity, classical integrable systems. On the other hand it is a classical subject in mathematics since the days of Riemann involving intricate structures. Any further one added to the impressive amount unraveled so far is nevertheless welcome to get a deeper understanding. According to a well established tradition, a natural temptation for physicists was to develop a manageable discretization. This was accomplished through the use of matrix models and their perturbative expansions in terms of decorated cell decompositions of surfaces, leading to a corresponding cellular complex in moduli space. This opened the way to a fascinating interplay between topological properties of moduli spaces and (a restricted class of) solutions 
of the integrable KP hierarchy as well as new insights on some non-perturbative aspects of quantum gravity in two dimensions.

On the other hand a new development occurred through the realization that cell decompositions of compact orientable surfaces are related in a natural way to arithmetic curves (defined over a finite extension of the rationals) as a consequence of a remarkable theorem of Belyi. While this was quite explicit in the work of mathematicians it was not perceived by physicists until the work by Shabat and Voevodsky. As a result, a new character entered the scene: the absolute Galois group of the maximal extension $\overline{\mathbb{Q}}$ over $\mathbb{Q}$, acting on cell decompositions (or fat graphs, or dessins d'enfants, a terminology borrowed from Grothendieck). The present note is designed to describe some aspects of these relations as well as connections with various subjects such as fuchsian triangle groups. This theory is admittedly still in its infancy but has the virtue of interlacing various disciplines from theoretical physics to combinatorics, finite group theory, automorphic forms, hypergeometric functions and number theory. It is therefore natural to explore at first some examples. This is our spirit here where we review the foundations and suggest a few non-trivial connections. It could be that techniques borrowed from physics might provide some light on the mysterious action of $\operatorname{Gal}(\overline{\mathbb{Q}} / \mathbb{Q})$. On the other hand one might hope that progress in this direction provides some help in physically motivated questions.

\section{Fuchsian Triangle Covering Groups for Curves over Number Fields}

Let $\mathscr{C}$ be a smooth projective algebraic curve defined over a number field $k$. By a theorem of Belyi [Be] there exists a non-constant rational function,

$$
\beta: \mathscr{C} \rightarrow \mathbb{P}_{1}(\mathbb{C})
$$

defined over a finite extension of $k$ and ramified only over the points 0,1 and $\infty$. We call such a function a Belyi function for $\mathscr{C}$. If $\mathfrak{h}=\mathfrak{h}^{+}$and $\mathfrak{h}^{-}$denote the upper and lower half planes respectively, then the connected components of $\beta^{-1}\left(\mathfrak{h}^{+}\right)$and $\beta^{-1}\left(\mathfrak{h}^{-}\right)$are the open cells of a triangulation of $\mathscr{C}$ with vertices forming the set $\beta^{-1}\{0,1, \infty\}$. If at each element of $\beta^{-1}\{1\}$ the order of ramification is assumed to be equal to 2 one can, following Grothendieck, Shabat, Voevodsky [Gr, VoSh, ShVo], consider the "dessin" $G$ on the curve $\mathscr{C}$ given by $\beta^{-1}[1, \infty]$. If necessary, on replacing the Belyi function $\beta$ by $4 \beta(1-\beta)$, we can always make this assumption. Returning to the general case, let $p, q, r$ be positive common multiples of the orders of ramification of $\beta$ over $0,1, \infty$, respectively. Suppose that $\left(\frac{1}{p}+\frac{1}{q}+\frac{1}{r}\right)<1$. Let $\triangle$ be the Fuchsian triangle group of signature $(p, q, r)$ acting on $\mathfrak{h}$. The $\triangle$-automorphic function $j$ given by the inverse of a Schwarz triangle function for a triangle with angles $\frac{\pi}{p}, \frac{\pi}{q}, \frac{\pi}{r}$ maps $\mathfrak{h}$ to $\mathbb{P}_{1}(\mathbb{C})$. One can normalise the function $j$ in such a way that the ramification order over $0,1, \infty$ are exactly $p, q, r$. On choosing a local branch of $\beta^{-1} \circ j$ that one continues analytically, and on applying the monodromy theorem, one sees that $j$ factorises as

$$
j=\beta \circ \Phi
$$

where $\Phi$ determines a (possibly) ramified covering of $\mathscr{C}$ by $\mathfrak{h}$ depending on $\beta$ and on $\triangle$. One has therefore the following generalization of the discussion in [ShVo], which appears again in Sect. 3 in the context of triangulations. 
Proposition 1. Let $\mathscr{C}$ be a smooth algebraic curve defined over a number field and let $\beta: \mathscr{C} \rightarrow \mathbb{P}_{1}(\mathbb{C})$ be a Belyi function. Let $p, q, r$ be finite positive common multiples of the orders of ramification of $\beta$ over $0,1, \infty$ respectively. Suppose that $\left(\frac{1}{p}+\frac{1}{q}+\frac{1}{r}\right)<1$. Let $\triangle$ be the cocompact Fuchsian triangle group of signature $(p, q, r)$ acting on $\mathfrak{h}$. There exists a holomorphic map

$$
\Phi: \mathfrak{h} \rightarrow \mathscr{C}
$$

ramified at most over the elements of $\beta^{-1}\{0,1, \infty\}$. The covering group $H$ of $\Phi$ is a subgroup of finite index in $\triangle$. There is therefore a Riemann surface isomorphism between $H \backslash \mathfrak{h}$ and $\mathscr{C}$.

The above discussion goes through equally well for non-cocompact triangle groups. Indeed, with $p=q=r=\infty$ we recover the known result that every smooth projective curve defined over $\overline{\mathbb{Q}}$ can be represented as a compactified quotient $\overline{H \backslash \mathfrak{h}}$ with subgroup $H$ of finite index in the principal subgroup of level 2 in $P S L_{2} \mathbb{Z}$. In the particular case where the ramifications of $\beta$ over 1 are all equal to 2 , one can choose $q=2$ and define $\beta$ and $H$ by means of the cartographic group of Grothendieck [Gr, ShVo].

Remarks. 1) The map $\Phi$ is the unramified universal covering map if and only if $p, q$, $r$ are the precise ramification numbers of $\beta$ in every point of $\beta^{-1}(0), \beta^{-1}(1), \beta^{-1}(\infty)$ respectively.

So the procedure we have described above can yield the universal covering map of $\Sigma$ if and only if there is a Belyi function $\beta$ on $\Sigma$ with constant ramification numbers in the fibres over $0,1, \infty$. We therefore can state the following consequence also noticed by Manfred Streit using a different method: The curve $\mathscr{C}$ has a universal covering group contained in a triangle group if a Belyi function exists on $\mathscr{C}$ defining a Galois covering $\mathscr{C} \rightarrow \mathbb{P}_{1}(\mathbb{C})$. By Galois covering, we mean that $H$ is a normal subgroup of $\triangle$.

2) The above construction contains two ambiguities. First there are many choices for the Belyi functions, and second there are infinitely many choices of the multiples of $p, q, r$ and hence for $\triangle=\triangle_{p, q, r}$ and for $j$. The problem arises therefore of obtaining more insight into the characterisation of these Belyi functions. Even if we fix a Belyi function $\beta$ on $\mathscr{C}$, we can realise it in many different ways as a natural projection,

$$
\mathscr{C}=\left(H_{p, q, r} \backslash \mathfrak{h}\right) \rightarrow \mathbb{P}_{1}(\mathbb{C})=\left(\triangle_{p, q, r} \backslash \mathfrak{h}\right),
$$

where $p, q, r$ denote common multiples of the respective ramification numbers of $\beta$ over $0,1, \infty$, and $H_{p, q, r}$ is the covering group of the covering map $\Phi$ of Proposition 1. One may ask how the different $H_{p, q, r}$ are related, and how they are related to the universal covering group of $\mathscr{C}$. Although these groups are not in general commensurable, we can at least prove a result of the following type. Under suitable normalisations and in the sense of Chabauty's topology, the $H_{p, q, r}$ converge with increasing $p, q, r$ to $H_{\infty, \infty, \infty}$, the subgroup already mentioned of $\triangle_{\infty, \infty, \infty}$ (the principal subgroup of level 2 in $P S L_{2}(\mathbb{Z})$ ). For the Chabauty convergence, see [Wo4]. It seems reasonable to restrict one's attention to this special case $H_{\infty, \infty, \infty}$ in order to obtain at least a partial uniqueness. On the one hand, we must in so doing delete the vertices of the triangulation when they may play a very important role, as we shall see in the next section. 


\section{Grothendieck Dessins and Shimura Varieties}

In this section we shall prove the following, announced in [CoWo2],

Theorem 1. Let $\mathscr{C}$ be a smooth algebraic curve defined over a number field and let $\beta: \mathscr{C} \rightarrow \mathbb{P}_{1}(\mathbb{C})$ be a Belyi function. With the notations of Proposition 1, Sect. 1 , the group $H$ determines an arithmetic group $\Gamma$ acting on $\mathfrak{h}^{t}$ of some positive integer $t$. The quotient $V \simeq \Gamma \backslash \mathfrak{h}^{t}$ is a Shimura variety. There exists an analytic injection $\mathfrak{h} \hookrightarrow \mathfrak{h}^{t}$ and a compatible group inclusion $H \hookrightarrow \Gamma$ inducing by passage to the quotient a non-trivial morphism defined over $\overline{\mathbb{Q}}$,

$$
\psi: \mathscr{C} \rightarrow V
$$

which sends the elements of $\beta^{-1}\{0,1, \infty\}$ onto points of complex multiplication by a subfield $\mathbb{K}$ of a cyclotomic field. The morphism $\psi$ and the field $\mathbb{K}$ depend on $\mathscr{C}, \beta$ and the conjugacy class of the chosen group $\triangle$ in $P S L_{2} \mathbb{R}$.

Proof. The group $\triangle$ is rigid, that is its conjugacy class is uniquely determined by its signature $(p, q, r)$, meaning that the presentation of $\triangle$ is given by

$$
\left\langle M_{1}, M_{2}, M_{3} \mid M_{1}^{p}=M_{2}^{q}=M_{3}^{r}=M_{1} M_{2} M_{3}=1\right\rangle .
$$

The statements of the theorem concerning the uniformisation of $\mathscr{C}$ by the subgroup $H$ of the triangle group $\triangle$ are given by Proposition 1 of Sect. 1 . The rest of the proof relies on the modular embedding method developed in [CoWo1]. Namely, let $\mathbb{L}$ be the field generated by the traces of the elements of $H$; then $H$ generates a quaternion algebra $B$ over $\mathbb{L}$ contained in $M_{2}(\mathbb{R})$. Let $\Gamma$ be the norm unit group in $B$. If $\mathbb{H}$ denotes the Hamiltonian quaternion algebra, there is an algebra isomorphism

$$
B \otimes_{\mathbb{Q}} \mathbb{R} \simeq M_{2}(\mathbb{R})^{t} \oplus \mathbb{H}^{d-t},
$$

where $d=[\mathbb{L}: \mathbb{Q}]$ and $t$ is the number of unramified infinite places of $\mathbb{L}$ with respect to $B$. By means of this isomorphism, one can define an action of $H$ and of $\Gamma$ on $\mathfrak{h}^{t}$ (see for example [Shi]). The inclusion of $H$ in the arithmetic group $\Gamma$ defines the modular embedding at the group level. At the level of the universal coverings, in [CoWo1] an analytic embedding $\mathfrak{h} \hookrightarrow \mathfrak{h}^{t}$ was constructed which commutes with the actions of $H$ (on $\mathfrak{h}$ ) and $\Gamma$ (on $\mathfrak{h}^{t}$ ). By passage to the quotient, one obtains a non-trivial morphism $\psi$ of $\mathscr{C} \simeq H \backslash \mathfrak{h}$ into $V=\Gamma \backslash \mathfrak{h}^{t}$. This morphism is defined over $\overline{\mathbb{Q}}$.

The space $\mathfrak{h}^{t}$ parametrises the abelian varieties of dimension $2 d$ with generalised complex multiplication by a purely imaginary extension $\mathbb{K}$ of $\mathbb{L}$. The field $\mathbb{K}$ is a subfield of the cyclotomic field $\mathbb{Q}\left(\exp \frac{2 \pi i}{l}\right)$, where $\frac{l}{2} \stackrel{\text { def }}{=}$ l.c.m. $(p, q, r)$, and the CM type of the abelian varieties is determined by the ramification of the infinite places of $B$ over $\mathbb{L}$. For these details the reader can consult Sect. 1 and the end of Sect. 3 of [CoWo1]. The images of the fixed points of $\triangle$ by the modular embedding are the CM points by Sect. 5 of [CoWo1] (p. 103 in particular). They correspond to the varieties which are isogenous to a product $A_{1} \times A_{2}$ of abelian varieties with complex multiplication by $\mathbb{K}$ in the strict sense, i.e. $2 \operatorname{dim}_{\mathbb{C}} A_{i}=[\mathbb{K}: \mathbb{Q}]$ for $i=1,2$. The images of the fixed point of $\triangle$ by the natural projection onto 
the quotient $\mathscr{C} \simeq H \backslash \mathfrak{h}$ form the set $\beta^{-1}\{0,1, \infty\}$, hence the statement of the theorem.

The reader will find some additional information about the modular embedding method of the above proof in Sect. 7.

Notice that for the Grothendieck dessins, $\psi$ sends not only the vertices of the dessin $G$ onto CM points, but also those of the dual dessin $G^{*}$ that one obtains by taking $\frac{1}{\beta}$ instead of $\beta$, and the points in the intersection of $G$ and $G^{*}$.

\section{Triangulations of Riemann Surfaces and Their Fields of Definition as Curves}

Voevodsky and Shabat [VoSh] have shown that a compact Riemann surface, considered as a smooth algebraic curve, is defined over a number field if and only if it can be triangulated by "equilateral" triangles. In this section we provide an account of the somewhat more general approach developed in the preprint [Wo1].

The essential property of compatibility between a given conformal structure and a given oriented triangulation $\mathfrak{T}$ of a compact Riemann surface $\Sigma$, in order that $\Sigma$ have the structure of a smooth algebraic curve $\mathscr{C}$ defined over a number field, is that for every edge $E$ of a triangle $T$ of $\mathfrak{T}$ there exist a reflection preserving $\mathfrak{T}$, fixing $E$, and mapping $T$ antiholomorphically onto the neighbouring triangle $T^{\prime}$ of $\mathfrak{T}$ sharing $E$. Such a reflection is not in general defined globally as a topological automorphism on $\Sigma$. Indeed to obtain a suitable global action one may either develop a combinatorial definition by means of the "cartographical group" of Grothendieck [G] and of others [VoSh, ShVo] discussed in a forthcoming section, or, as we do in the present section, pass to a simply connected, but possibly ramified covering $\tilde{\Sigma}$ of $\Sigma$. The triangulation $\mathfrak{T}$ of $\Sigma$ lifts to a triangulation $\tilde{\mathcal{T}}$ of $\tilde{\Sigma}$ and the appropriate global action on $\tilde{\Sigma}$ of a reflection group is provided by well-defined reflections in the sides of the elements of $\tilde{\mathfrak{T}}$. These reflections generate an extension of index 2 of a triangle group. The group of the possibly ramified cover $\tilde{\Sigma}$ of $\Sigma$ is a subgroup of finite index in this triangle group. When $\tilde{\Sigma}=\mathfrak{h}$, using the components of $\beta^{-1}\left(\mathfrak{h}^{+}\right), \beta^{-1}\left(\mathfrak{h}^{-}\right)$as triangulation, we thus recover Proposition 1.

Let us now be more specific about the properties that the oriented triangulation $\mathfrak{T}$ of the compact Riemann surface $\Sigma$ underlying a smooth algebraic curve $\mathscr{C}$ must have in order to assure that the field of definition of the curve is a number field. Let $\mathfrak{T}$ consist of $2 N$ open cells $T=T_{n}^{+}$(positive orientation) and $T_{m}^{-}$(negative orientation) with $n, m=1, \ldots, N$ homeomorphic to euclidean triangles, and let $\tilde{\Sigma}$ be simply connected covering space of $\Sigma$, possibly ramified over the vertices of the triangulation of $\Sigma$. One may even allow $\tilde{\Sigma}$ to be a covering space of $\Sigma$ minus some of the vertices: we refer then to a logarithmic ramification over these vertices. In any case, the triangulation $\mathfrak{T}$ lifts to a triangulation $\tilde{\mathfrak{T}}$ of $\tilde{\Sigma}$ by (in general infinitely many) open cells $\tilde{T}=\tilde{T}_{\nu}^{+}$and $\tilde{T}_{\mu}^{-}, \nu, \mu=1,2, \ldots$ We will simply call all these open cells "triangles."

Definition. We call $(\mathfrak{T}, \tilde{\Sigma})$ a "covered symmetric triangulation" of $\Sigma$ if it satisfies the following properties: For a $\tilde{T}$ among the elements of $\tilde{\mathfrak{T}}$ there exist three reflections $\sigma_{j}$ in the sides $e_{j}, j=1,2,3$ of $\tilde{T}$ such that :

a) the $\sigma_{j}$ are homeomorphisms of $\tilde{\Sigma}$ onto itself, preserving the triangulation by the $\tilde{T}_{\nu}^{+}, \tilde{T}_{\mu}^{-}$, 
b) each $\sigma_{j}$ maps $\tilde{T}$ onto its neighbour sharing the edge $e_{j}$ with $\tilde{T}$. Moreover, $\left.\sigma_{j}\right|_{e_{j}}$ is the identity and $\sigma_{j}^{2}=1$ for $j=1,2,3$,

c) the action of the $\sigma_{j}$ on the triangles changes the orientation, i.e. for all $\tilde{T}_{\nu}^{ \pm}$and $j$ there is a $\tilde{T}_{\mu}^{\mp}$ such that $\sigma_{j} \tilde{T}_{\nu}^{ \pm}=\tilde{T}_{\mu}^{\mp}$,

d) the covering map $\Phi: \tilde{\Sigma} \rightarrow \Sigma$ is holomorphic and the $\sigma_{j}: \tilde{\Sigma} \rightarrow \tilde{\Sigma}$ are antiholomorphic maps.

We may now formulate the following consequence of Belyi's theorem:

Theorem 2. A smooth projective algebraic curve over the complex numbers is defined over a number field if and only if, as a compact Riemann surface, it has a covered symmetric triangulation.

Before passing to the proof of this theorem, we note that, for a given algebraic curve defined over $\overline{\mathbb{Q}}$ we have infinitely many possible covered symmetric triangulations. But in the opposite direction, any such triangulation determines uniquely the conformal structure of the underlying Riemann surface and therefore of the algebraic curve up to isomorphism. Even more is true: as only property d) of the above definition involves the conformal structures of $\Sigma, \tilde{\Sigma}$, and the analytic properties of $\Phi, \sigma_{\gamma}$, $j=1,2,3$, we may use the rest of the definition just as well for compact orientable two-manifolds on replacing d) by the hypothesis:

$\left.\mathrm{d}^{\prime}\right)$ For any $\tau$ in the group generated by $\sigma_{1}, \sigma_{2}, \sigma_{3}$ in Aut $\tilde{\Sigma}$, the property $\tau \tilde{T}=\tilde{T}$ implies $\tau=I d$.

We use the nomenclature "covered symmetric triangulation" also in this case. Under this variant of the definition, the following has been shown in [Wo1] by some straightforward topological arguments similar to those of the proof of Theorem 2 below:

Proposition 2. Let $\Sigma$ be a compact oriented two-manifold with a covered symmetric triangulation. Then there are conformal structures on $\tilde{\Sigma}$ and $\Sigma$ unique to biholomorphic mappings such that $\Phi: \tilde{\Sigma} \rightarrow \Sigma$ is holomorphic and the $\sigma_{j}: \tilde{\Sigma} \rightarrow \tilde{\Sigma}$ are antiholomorphic. By Theorem 2, this determines uniquely the structure of $\Sigma$ as an algebraic curve defined over $\overline{\mathbb{Q}}$.

Proof of Theorem 2. Proof of the "only if" part; from Belyi functions to triangulations: Let $\mathscr{C}$ be a smooth projective algebraic curve defined over $\overline{\mathbb{Q}}$ and $\Sigma$ be its underlying Riemann surface. By Belyi's theorem [Be] there is a Belyi function $\beta: \Sigma \rightarrow \mathbb{P}_{1}(\mathbb{C})$ ramified only over the points $0,1, \infty$. Let $p, q, r$ be either $\infty$ or natural numbers divisible by all ramification numbers of $\beta$ over $0,1, \infty$ respectively. Consider the triangle group $\triangle$ of signature $(p, q, r)$ acting as a discontinuous group of holomorphic automorphisms on $\tilde{\Sigma}=\overline{\mathbb{C}}, \mathbb{C}$, or $\mathfrak{h}$ according to whether $\left(\frac{1}{p}+\frac{1}{q}+\frac{1}{r}\right)$ is $>1,=1$, or $<1$. The other cases being completely analogous, we concentrate on the hyperbolic case $\left(\frac{1}{p}+\frac{1}{q}+\frac{1}{r}\right)<1$. Fix a fundamental domain for the action of $\triangle$ on $\tilde{\Sigma}=\mathfrak{h}$. This domain is the union of two hyperbolic triangles with angles $\frac{\pi}{p}$, $\frac{\pi}{q}, \frac{\pi}{r}$. Fix one of these triangles, $\mathscr{T}_{0}^{+}$. The hyperbolic reflections $\sigma_{1}, \sigma_{2}, \sigma_{3}$ in the 
sides of $\mathscr{T}_{0}^{+}$generate a group $\triangle_{R}$ with defining relations

$$
\sigma_{1}^{2}=\sigma_{2}^{2}=\sigma_{3}^{2}=\left(\sigma_{1} \sigma_{2}\right)^{p}=\left(\sigma_{2} \sigma_{3}\right)^{q}=\left(\sigma_{3} \sigma_{1}\right)^{r}=1,
$$

where if, for example, $p=\infty$, the relation $\left(\sigma_{1} \sigma_{2}\right)^{p}=1$ is omitted. The group $\triangle$ consists of all products of an even number of $\sigma_{j}$ 's and is a normal subgroup of index 2 in $\triangle_{R}$ generated by

$$
M_{1}=\sigma_{1} \sigma_{2}, \quad M_{2}=\sigma_{2} \sigma_{3}, \quad M_{3}=\sigma_{3} \sigma_{1},
$$

and with defining relations as given in Sect. 2,

$$
M_{1}^{p}=M_{2}^{q}=M_{3}^{r}=M_{1} M_{2} M_{3}=1 .
$$

Let $\tilde{\mathfrak{T}}$ be the triangulation (subordinate to a tessellation of $\mathfrak{h}$ ) comprising the $\mathscr{T}_{\nu}^{+}$, given by all $\triangle$-images of $\mathscr{T}_{0}^{+}$, together with the $\mathscr{T}_{\mu}^{-}$, given by all $\triangle$-images of $\sigma_{2} \mathscr{T}_{0}^{+}$, so that the conditions of the definition of a covered symmetric triangulation are satisfied in as much as they concern $\mathfrak{h}$. The remarks preceding Proposition 1 of Sect. 1 show that there exists a holomorphic map $\Phi: \mathfrak{h} \rightarrow \Sigma$ ramified only in the vertices of the triangulation and inducing a finite triangulation of $\Sigma$ by the $\Phi$-images of the $\mathscr{T}_{\nu}^{+}, \mathscr{T}_{\mu}^{-}$. The image of the triangulation $\tilde{\mathfrak{T}}$ of $\mathfrak{h}$ under the map $\Phi$ is a finite triangulation $\mathfrak{T}$ of $\Sigma$ by triangles $T_{n}^{+}, T_{m}^{-}, n, m=1, \ldots, N$, where $N$ is the mapping order of the Belyi function $\beta$, that is the number of its sheets. The $2 N$ triangles $T_{n}^{+}$, $T_{m}^{-}$are just the connected components of $\beta^{-1}\left(\mathfrak{h}^{+}\right), \beta^{-1}\left(\mathfrak{h}^{-}\right)$.

Proof of the "if" part; from triangulations to Belyi functions. Let $(\mathfrak{T}, \tilde{\Sigma})$ be a covered symmetric triangulation of a Riemann surface $\Sigma$. Let $\triangle_{R}$ be the group of automorphisms of $\tilde{\Sigma}$ generated by the $\sigma_{j}, j=1,2,3$ satisfying a), b), c) in the definition of such a triangulation. As $\tilde{\Sigma}$ is connected, the group $\triangle_{R}$ acts transitively on the set of triangles in $\tilde{\mathfrak{T}}$. For the purposes of exposition, assume that the covering map $\Phi: \tilde{\Sigma} \rightarrow \Sigma$ has finite ramification numbers. Then the vertices of a choice of $\tilde{T}$ as in the definition are contained in $\tilde{\Sigma}$. Now, we can join $\tilde{T}$ to any other element of $\tilde{T}$ by a finite path avoiding the vertices of this triangulation. Let $\tilde{T}$ denote the closure of $\tilde{T}$. This path can be covered by a finite number of $\triangle_{R}$-images, $\bar{T}$, $\sigma_{j} \bar{T}, \sigma_{j} \sigma_{k} \bar{T}, \ldots, \tau \bar{T}$, for some $\tau \in \triangle_{R}$, since for every $\sigma \in \triangle_{R}$ the triangle $\sigma \tilde{T}$ has as neighbours the $\sigma \sigma_{j} \tilde{T}, j=1,2,3$. By the finiteness of the triangulation of $\Sigma$, for a given vertex of $\bar{T}$ there is a finite positive integer $p$ such that the set of closed triangles filling up a neighbourhood of this vertex has elements, $\bar{T}, \sigma_{i} \bar{T}$, $\sigma_{\imath} \sigma_{j} \bar{T}, \sigma_{i} \sigma_{j} \sigma_{i} \bar{T}, \ldots,\left(\sigma_{i} \sigma_{j}\right)^{p-1} \sigma_{i} \bar{T}=\sigma_{j} \bar{T}$ for some $i, j=1,2,3$ say $i=1, j=2$. In this sequence of neighbouring triangles the sign changes at each step so that we cannot have, $\left(\sigma_{1} \sigma_{2}\right)^{p-1} \tilde{T}=\sigma_{2} \tilde{T}$. Therefore we have, $\left(\sigma_{1} \sigma_{2}\right)^{p-1} \sigma_{1} \tilde{T}=\sigma_{2} \tilde{T}$ and $\left(\sigma_{1} \sigma_{2}\right)^{p} \tilde{T}=\tilde{T}$. The transformation $\left(\sigma_{1} \sigma_{2}\right)^{p}$ is the identity since it is a holomorphic transformation fixing the three vertices of $\bar{T}$ (for the compact oriented 2-manifolds in Proposition 2 of the present section, this argument must be replaced by condition $\left.\mathrm{d}^{\prime}\right)$ ). In the same way we prove the existence of finite positive integers $q, r$ with $\left(\sigma_{2} \sigma_{3}\right)^{q}=\left(\sigma_{3} \sigma_{1}\right)^{r}=1$. Since $\tilde{\Sigma}$ is simply connected, these local relations coming from closed loops around a fundamental domain for $\triangle_{R}$ are sufficient to give the presentation, already encountered in the "only if" part of the proof, of $\triangle_{R}$ (see for example, $[\mathrm{P}, \mathrm{Ge}, \mathrm{Beh}])$ :

$$
\triangle_{R}=\left\langle\sigma_{1}, \sigma_{2}, \sigma_{3} \mid \sigma_{1}^{2}=\sigma_{2}^{2}=\sigma_{3}^{2}=\left(\sigma_{1} \sigma_{2}\right)^{p}=\left(\sigma_{2} \sigma_{3}\right)^{q}=\left(\sigma_{3} \sigma_{1}\right)^{r}=1\right\rangle .
$$


By the rigidity of triangle groups, the group $\triangle_{R}$ is the index 2 extension of the triangle group $\triangle$ of signature $(p, q, r)$, with the presentation also given in the "only if" part of the proof, the number $\left(\frac{1}{p}+\frac{1}{q}+\frac{1}{r}\right)$ determining $\tilde{\Sigma}$. For convenience consider the case where $\tilde{\Sigma}=\mathfrak{h}$. As the triangulation of $\mathfrak{h}$ is induced by the triangulation of $\Sigma$, the covering group $H$ of $\tilde{\Sigma} \rightarrow \Sigma$ must preserve the triangulation of $\tilde{\Sigma}$. If $p, q, r$ are mutually distinct, we have $H \subset \triangle$ and therefore $\Sigma \simeq H \backslash \tilde{\Sigma}$ is defined over $\overline{\mathbb{Q}}$ (this fact is well-known, but we shall come back to it in Remark 2) following the proof). If some of the $p, q, r$ coincide we need a slightly different argument: For every $\gamma \in H$ there is a $\delta \in \triangle$ with either $\gamma \tilde{T}=\delta \tilde{T}$ or $\gamma \tilde{T}=\delta \sigma_{3} \tilde{T}$, depending on whether $\gamma$ changes the signs labelling the elements of $\tilde{\mathfrak{T}}$ or not. In other words, either $\delta^{-1} \gamma$ or $\sigma_{3} \delta^{-1} \gamma$ is an automorphism of both $\mathfrak{h}$ and $\tilde{T}$. In the first case, $\delta^{-1} \gamma$ is therefore either the identity or (only in the case $p=q=r$ ) it defines a rotation of $\tilde{T}$ of order 3 and belongs to an extension $\bar{\Delta}$ of $\triangle$ which is again a triangle group (of signature $(2,3,2 p$ ) and index 6 over $\triangle$ ). In the sign-changing case, the triangle $\sigma_{3} \tilde{T}$ is both a holomorphic and a reflected image of $\tilde{T}$. This is possible if and only if the signature of $\Delta$ has the special form $(p, q, q)$, and in this case $\delta^{-1} \gamma$ again belongs to an extension of $\triangle$, namely a triangle group $\bar{\triangle}$ of signature $(2 p, q, 2)$. In any case, the covering group of $\Phi$ is

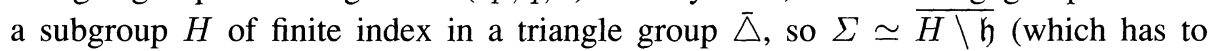
be compactified in the usual way if $\Gamma$ has cusps). Once again such a quotient has the structure of an algebraic curve defined over $\overline{\mathbb{Q}}$. This completes the proof of the theorem.

Remark. 1) In the "if" part of the above proof, the complex structure of the Riemann surface $\Sigma$ is completely determined by the covering group $H$, and hence by the triangulation.

2) For the convenience of the reader we recall Belyi's argument [Be], used at several points in the above proof, whereby $\mathscr{C} \simeq H \backslash \mathfrak{h}$ is defined over $\overline{\mathbb{Q}}$ if $H$ is a subgroup of finite index in a cocompact triangle group $\triangle$ or equivalently (by the above results) if a Belyi function on $\mathscr{C}$ exists. Let $(\mathscr{C}, \beta)$ be defined over a field $\mathbb{K} \subset \mathbb{C}$, that is $\mathscr{C}$, that is $\mathscr{C}$ and $\beta$ are given by equations with coefficients in $\mathbb{K}$. Any embedding $\alpha: \mathbb{K} \hookrightarrow \mathbb{C}$ defines (by its action on coefficients) a conjugate curve $\mathscr{C}^{\alpha}$ and induces from the Belyi function $\beta$ on $\mathscr{C}$, a morphism $\beta^{\alpha}: \mathscr{C}^{\alpha} \rightarrow \mathbb{P}_{1}(\mathbb{C})$. The morphism $\beta^{\alpha}$ is also a Belyi function with the same ramification numbers and of the same mapping order $N$ as $\beta$. Hence $\mathscr{C} \simeq H \backslash \mathfrak{h}$ and $\mathscr{C}^{\alpha} \simeq H^{\alpha} \backslash \mathfrak{h}$ with two subgroups $H$ and $H^{\alpha}$ of the same rigid triangle group $\triangle$. (By "rigid" is understood "uniquely determined up to conjugation in $P S L_{2}(\mathbb{R})$ by its presentation in terms of generators and relations"). Furthermore, the groups $H$ and $H^{\alpha}$ have the same index $N$ in $\triangle$, and there are only finitely many subgroups of this index in $\triangle$ ([MKS], p. 102, Exercise 19), so we can obtain only finitely many non-isomorphic curves $\mathscr{C}^{\alpha}$ by this procedure. If $\mathscr{C}$ is an elliptic curve, by inspection of the modular invariant we see that $\mathscr{C}$ must be defined over a number field. If $\mathscr{C}$ has a higher genus, we have to apply Theorem 4 of [We] and to control whether, for isomorphisms $f_{\alpha \beta}: \mathscr{C}^{\beta} \rightarrow \mathscr{C}^{\alpha}$ between such conjugate curves, we shall obtain,

$$
f_{\alpha \beta} \circ f_{\beta \gamma} \circ f_{\gamma \alpha}=i d
$$

This condition is easily seen to be satisfied for such isomorphisms $f_{\alpha \beta}$ at least if we combine them with one of the finitely many automorphisms of $\mathscr{C}^{\alpha}$. 
We end this section by observing that, in the "if" part of the above proof, we can of course go back from the quotient space to the Belyi function: Let $j$ be the modular function for the triangle group $\bar{\triangle} \supset$ and $H(z)$ the orbit of $z$ under $H$, then

$$
\beta(H(z)) \stackrel{\text { def }}{=} j(z)
$$

defines a Belyi function on the curve $\mathscr{C}$ whose underlying Riemann surface is $\Sigma$. The covered symmetric triangulation of $\Sigma$, whose elements are the connected components of $\beta^{-1}\left(\mathfrak{h}^{+}\right)$and $\beta^{-1}\left(\mathfrak{h}^{-}\right)$, is the $\Phi$-image of the triangulation of $\mathfrak{h}$ by the usual fundamental domains of $\bar{\triangle}_{R}$.

We have therefore,

Proposition 3. Let $\mathscr{C}$ be a smooth algebraic curve defined over a number field. Every Belyi function on $\mathscr{C}$ defines a covered symmetric triangulation, and every covered symmetric triangulation has a subtriangulation coming from a Belyi function.

\section{Fermat Curves: An Example}

Consider the covered symmetric triangulations obtained in Sect. 3. By applying a combination of inverse triangle functions and Schwarz-Christoffel maps, we can find a biholomorphic mapping of any curvilinear triangle onto any euclidean triangle. In particular we can replace our triangles by equilateral triangles in the spirit of [VoSh]. Yet there is a different and, in the context of Theorem 2 of Sect. 3, more natural way to construct "equilateral " triangulations. Namely, let $\Sigma$ be the Riemann surface underlying a smooth projective algebraic curve $\mathscr{C}$ defined over $\overline{\mathbb{Q}}$ with Belyi function $\beta$. We can choose an integer $M>3$ as a common multiple for all the ramification numbers of $\beta$. We may then apply the construction of the covered symmetric triangulation of Sect. 3 using the triangle group $\triangle=\triangle_{M}$ of signature $(M, M, M)$ acting on $\mathfrak{h}$. This gives rise to an equilateral hyperbolic triangulation $\tilde{T}_{M}$ of $\tilde{\Sigma}=\mathfrak{h}$ by hyperbolic triangles of angles $\frac{\pi}{M}, \frac{\pi}{M}, \frac{\pi}{M}$. Notice however that even if $\Sigma$ has genus greater than 1 , as the map $\Phi: \mathfrak{h} \rightarrow \Sigma$ is not in general the universal covering map, the hyperbolic metric on $\Sigma$ is not obtained from the image of this covering triangulation under $\Phi$ in the same way as it is from the equilateral triangulation in [VoSh]. Nevertheless, this triangulation enables one to see that any curve defined over $\overline{\mathbb{Q}}$ shares a common ramified finite covering curve with a Fermat curve. Indeed, let $F_{M}$ be the Fermat curve of exponent $M$, so that its affine equation is $x^{M}+y^{M}=1$ and its universal covering group is the commutator subgroup [ $\triangle, \triangle$ ] of $\triangle$. Now $\Sigma=H \backslash \mathfrak{h}$, where $H$ is the covering group of $\Phi$. Both $H$ and $[\triangle, \triangle]$ are of finite index in $\triangle$. By taking a normal subgroup $\Upsilon$ of $\triangle$ in their intersection, we obtain a finite ramified covering curve $Y=\Upsilon \backslash \mathfrak{h}$ of both $\Sigma$ and $F_{M}$. This induces a correspondence between $\Sigma$ and $F_{M}$. All the covered symmetric triangulations of $Y$, $\Sigma$ and $F_{M}$ are images of the same triangulation $\tilde{T}_{M}$ of $\mathfrak{h}$.

Restricting our attention now to $F_{M}$, let us recall how to calculate its genus $g_{M}$. Using the Riemann-Hurwitz formula (see for example [Cn, p. 143]) we have $g_{M}=1-M+b / 2$ with $b=\sum\left(e_{i}-1\right)$, where the $e_{\imath}$ range over the exponents of uniformisation at the branch points. One writes

$$
y^{M}=1-x^{M}=(-1)^{M}(x-1)\left(x-\zeta_{M}\right) \ldots\left(x-\zeta_{M}^{M-1}\right) .
$$


for $j=0, \ldots, M-1$, a branch point over $x=\zeta^{j}$ contributes $M-1$ to $b$. Therefore $g_{M}=(M-1)(M-2) / 2$.

It is easy to check that

$$
\triangle /[\triangle, \triangle] \simeq \mathbb{Z} / M \mathbb{Z} \oplus \mathbb{Z} / M \mathbb{Z}
$$

Indeed, the group acts on $F_{M}$ by the automorphisms

$$
\begin{aligned}
\varrho(x, y) & =\left(\zeta_{M} x, y\right), \\
\varrho^{\prime}(x, y) & =\left(x, \zeta_{M} y\right),
\end{aligned}
$$

where $\zeta_{M}=\exp \left(\frac{2 \pi i}{M}\right)$. As the fundamental region of $\triangle$ in $\mathfrak{h}$ consists of 2 triangles, it is clear that $\Phi$ induces on $F_{M}$ a triangulation by $2 M^{2}$ triangles, with $3 M^{2}$ common edges, and each of the $3 M$ vertices common to $2 M$ triangles. One may cross-check that one has the correct value for $g_{M}$. The $2 M^{2}$ triangles of this covered symmetric triangulation on $F_{M}$ are the connected components of the pre-images of $\mathfrak{h}^{+}$and $\mathfrak{h}^{-}$by the Belyi function $\beta_{M}(x, y) \mapsto x^{M}$. The 1-skeleton of this triangulation even forms a Grothendieck dessin $G$ (but not for $\beta_{M}$ !) on $F_{M}$. To see this, use the fact that there is an extended triangle group $\triangle$ containing $\triangle$ and with signature $(2,3,2 M)$ whose modular $\left(j\right.$-)function induces a clean Belyi function on $F_{M}$.

In the context of Theorem 1 of Sect. 2, in the cases $M=4,5,6,7,8,9,12,15$, the group $\triangle$ is arithmetic [Ta], so that the morphism $\psi$ of the curve $\mathscr{C}$ to a Shimura variety $V$ is the identity and $\mathscr{C}=V$ is a Shimura curve which obviously has an infinity of $\mathrm{CM}$ points. For the other values of $M$, the morphism $\psi$ sends at least the vertices of the triangulation of $\mathscr{C}$ onto the $\mathrm{CM}$ points of a Shimura variety $V$ of complex dimension greater than 1 . One can determine explicitly the field $\mathbb{K}$ and the CM type of the simple factors of the abelian varieties corresponding to the vertices. On p. 107 of [CoWo1] we treated the example $M=10$.

Fermat curves will also be central to our discussion of rational triangular billiards in Sect. 6.

\section{The Cartographic Group}

The abstract cartographic group $\mathscr{C}_{2}[\mathrm{Gr}]$ may be defined by the presentation

$$
\left\langle r_{0}, r_{1}, r_{2} \mid r_{0}^{2}=r_{1}^{2}=r_{2}^{2}=\left(r_{0} r_{2}\right)^{2}=1\right\rangle \text {. }
$$

We begin by supplementing the discussion of [VoSh, ShVo and Gr], in which it is indicated how a connected (finite) cell complex gives rise to a representation of this group. Recall (see for example [RoSa, p. 26, Dic, pp. 244-248]), that an abstract (finite) simplicial complex $S$ consists of a finite set $J_{0}(S)$ (the vertices), together with a family $J=J(S)$ of subsets of $J_{0}(S)$ (the simplexes), such that if $j \subset j^{\prime} \in J$ then $j \in J$. The abstract simplicial complex $S$ can be realised as a simplicial complex in $\mathbb{R}^{\left|J_{0}\right|-1}$ (see for example [LuWe, p. 17]). A simplex consisting of $n+1$ distinct vertices is called an $n$-simplex. We wish to consider such abstract information coming from a connected (finite) cell complex $K$ which satisfies certain regularity assumptions. As we shall indicate at the end of this section, when we consider the formal context of abstract cell complexes as treated for example in [AhlSa, Ch. 1§7], these regularity assumptions can be weakened for the definition of the oriented cartographic group (see also [ShVo, p. 207, 1.4]). It is instructive to work first in a non-oriented context. 
A connected (finite) cell complex $K$ gives a finite set $V=J_{0}(K)$ (the vertices) and collections $E=J_{1}(K)$ (the edges) and $F=J_{2}(K)$ (the faces) of subsets of $J_{0}(K)$. The edges consist of 2-element subsets of $J_{0}(K)$. The faces $F$ consist of $n$-element subsets, $n \geq 2$, of $J_{0}(K)$ of the form $\left\{v_{1}, \ldots, v_{n}\right\}$ with $e_{\imath}=\left\{v_{i}, v_{i+1}\right\}$, $i=1, \ldots, n-1, e_{n}=\left\{v_{n}, v_{1}\right\} \in E$. If $n=3$ for every face then all the three distinct 2-element subsets of a face must be edges of the complex: hence every face is a 2 simplex and we recover an abstract simplicial complex. The flag set $\mathscr{F}=\mathscr{F}(K)$ of $K$ consists of triples $(v, e, f)$ with $v \in V, e \in E, f \in F$ such that $u=\{v\} \subset e \subset f$. Let the $\pi_{q}: \mathscr{F}(K) \rightarrow J_{q}(K), q=0,1,2$ be the corresponding projections. We make the following regularity assumptions (compare with [ShVo, p. 200 0.1]) ((i) follows from the above definitions):

(i) each edge $e$ contains exactly 2 vertices, $v=v(e)$ and $v^{\sigma}=v^{\sigma}(e), \sigma^{2}=1$,

(ii) a given face $f$ has each of its vertices $v$ lying on exactly 2 of its edges, $e=e(v \subset f)$ and $e^{\sigma}=e^{\sigma}(v \subset f), \sigma^{2}=1$,

(iii) each edge $e$ lies on exactly 2 faces, $f=f(e)$ and $f^{\sigma}=f^{\sigma}(e), \sigma^{2}=1$.

This enables us to define the following operations on $\mathscr{F}$,

$$
\begin{aligned}
\sigma_{0}:(v, e, f)=(v(e), e, f) & \mapsto\left(v^{\sigma}, e, f\right)=\left(v^{\sigma}(e), e, f\right), \\
\sigma_{1}:(v, e, f)=(v, e(v \subset f), f) & \mapsto\left(v, e^{\sigma}, f\right)=\left(v, e^{\sigma}(v \subset f), f\right), \\
\sigma_{2}:(v, e, f)=(v, e, f(e)) & \mapsto\left(v, e, f^{\sigma}\right)=\left(v, e, f^{\sigma}(e)\right) .
\end{aligned}
$$

Clearly,

$$
\sigma_{0} \sigma_{2}:(v, e, f)=(v(e), e, f(e)) \mapsto\left(v^{\sigma}, e, f^{\sigma}\right)=\left(v^{\sigma}(e), e, f^{\sigma}(e)\right)
$$

so that

$$
\sigma_{0}^{2}=\sigma_{1}^{2}=\sigma_{2}^{2}=\left(\sigma_{0} \sigma_{2}\right)^{2}=1
$$

We now notice that the connectedness of $K$ requires the action of the $\sigma_{i}, i=0,1,2$ on $\mathscr{F}$ to be transitive. We denote by $\mathscr{C}_{2}{ }^{K}$ the representation of $\mathscr{C}_{2}$ defined by $r_{i} \mapsto \sigma_{i}, i=0,1,2$. Moreover, for all $p, q \in\{0,1,2\}$ and all $L \in \mathscr{F}$ one has $\pi_{q}\left(\sigma_{p} \circ L\right)=\pi_{q}(L)$ if and only if $p \neq q$.

Now, we assume the existence of an orientation on $\mathscr{F}$, that is a function $o: \mathscr{F} \rightarrow\{+1,-1\}$ satisfying for all $q \in\{0,1,2\}, L \in \mathscr{F}$,

$$
o\left(\sigma_{q} \circ L\right)=-o(L) \text {. }
$$

In view of the above remarks, given $v \in V, e \in E,\{v\} \subset e$, the two faces $f$ with $e \subset f$ may be distinguished by the orientation: one writes $f=f_{0}(v, e)$ if $(v, e, f)$ is positively oriented, and $f=f_{1}(v, e)$ if $(v, e, f)$ is negatively oriented. Using the orientation in an analogous way, given $v \in V, f \in F$ with $v \subset f$ we define $e_{0}(v, f)$ and $e_{1}(v, f)$, given $e \subset f$ we define $v_{0}(e, f)$ and $v_{1}(e, f)$. Therefore, starting with a flag $L=(v, e, f)$ we have $v=v_{\imath}(e, f), e=e_{i}(v, f), f=f_{i}(e, v)$ where, depending on whether or not $L$ is positively or negatively oriented, $i=0$ or 1 respectively. Let $\sigma \in \operatorname{Perm}\{0,1\} \simeq S_{2}$ be the transposition $\sigma=(0,1)$.

We may now rewrite the operations $\sigma_{q}, q=0,1,2$, in a more convenient form as follows:

$$
\sigma_{0}:(v, e, f)=\left(v_{i}(e, f), e, f\right) \mapsto\left(v^{1}, e, f\right)=\left(v_{\sigma(\imath)}(e, f), e, f\right)
$$


where, as the orientation has been reversed, we have

$$
v^{1}=v_{\sigma(i)}(e, f), \quad e=e_{\sigma(i)}\left(v^{1}, f\right), \quad f=f_{\sigma(i)}\left(v^{1}, e\right) .
$$

Similarly,

$$
\sigma_{1}:(v, e, f) \mapsto\left(v, e^{1}, f\right)
$$

where, if $e=e_{\imath}(v, f)$, we have

$$
\begin{gathered}
e^{1}=e_{\sigma(i)}(v, f), \quad v=v_{\sigma(\imath)}\left(e^{1}, f\right), \quad f=f_{\sigma(i)}\left(v, e^{1}\right), \\
\sigma_{2}(v, e, f) \mapsto\left(v, e, f^{1}\right),
\end{gathered}
$$

where, if $f=f_{i}(v, e)$, we have

$$
f^{1}=f_{\sigma(i)}(v, e), \quad v=v_{\sigma(i)}\left(e, f^{1}\right), \quad e=e_{\sigma(i)}\left(v, f^{1}\right) .
$$

Using this notation, it is easy to compute the effect of $\varrho_{0}=\sigma_{2} \sigma_{1}, \varrho_{1}=\sigma_{0} \sigma_{2}$, $\varrho_{2}=\sigma_{1} \sigma_{0}$ on $L=(v, e, f) \in \mathscr{F}, v=v_{i}(e, f), e=e_{i}(v, f), f=f_{i}(v, e)$, where $i=0$ if $o(L)=1$ and $i=1$ if $o(L)=-1$. Namely,

$$
\begin{aligned}
\varrho_{0}:(v, e, f) & \mapsto\left(v, e^{1}, f^{1}\right), \\
e^{1}=e_{\sigma(i)}(v, f)=e_{i}\left(v, f^{1}\right), \quad f^{1} & =f_{i}\left(v, e^{1}\right), \quad v=v_{i}\left(e^{1}, f^{1}\right), \\
\varrho_{1}:(v, e, f) & \mapsto\left(v^{1}, e, f^{1}\right), \\
f^{1}=f_{\sigma(i)}(v, e)=f_{\imath}\left(v^{1}, e\right), \quad v^{1} & =v_{i}\left(e, f^{1}\right), \quad e=e_{\imath}\left(v^{1}, f^{1}\right), \\
\varrho_{2}:(v, e, f) & \mapsto\left(v^{1}, e^{1}, f\right), \\
v^{1}=v_{\sigma(i)}(e, f)=v_{i}\left(e^{1}, f\right), \quad e^{1} & =e_{i}\left(v^{1}, f\right), \quad f=f_{\imath}\left(v^{1}, e^{1}\right) .
\end{aligned}
$$

If the valency of a vertex is the number of edges containing it, and the valency of a face is the number of edges it contains, it is clear by inspection of the above expressions, that if for $L=(v, e, f) \in \mathscr{F}$ we let $\nu_{0}(L)$ be the valency of $v$ and $\nu_{2}(L)$ be the valency of $f$, we have

$$
\begin{aligned}
\varrho_{0}^{\nu_{0}(L)}(L) & =L, \\
\varrho_{1}^{2}(L) & =L, \\
\varrho_{2}^{\nu_{2}(L)}(L) & =L .
\end{aligned}
$$

Notice that an edge always has "valency" 2.

We denote by $\mathscr{C}_{2}^{+, K}$ the oriented cartographic group, that is the subgroup of index 2 of $\mathscr{C}_{2}^{K}$ generated by the words in the $\sigma_{i}, i=0,1,2$ of even length. Clearly the $\varrho_{i}$, $i=0,1,2$ are generators of $\mathscr{C}_{2}^{+, K}$ and satisfy $\varrho_{2} \varrho_{1} \varrho_{0}=1$.

We now construct an abstract simplicial complex $S=S(K)$ which may be thought of as a (barycentric) subdivision of $K$. We start with $J_{0}(K)$, then for every edge $e \in J_{1}(K)$ we add a vertex $v_{e} \notin J_{0}(K)$ and for every face $f \in J_{2}(K)$ we add a vertex $v_{f} \notin J_{0}(K)$ with $v_{f} \neq v_{e}$ for any $e \in J_{1}(K)$. We require $S$ to be the (finite, abstract) simplicial complex with vertices $J_{0}(S)=\left\{v \mid v \in J_{0}(K)\right\} \cup\left\{v_{e} \mid e \in\right.$ $\left.J_{1}(K)\right\} \cup\left\{v_{f} \mid f \in J_{2}(K)\right\}$, edges $J_{1}(S)$ the 2-element subsets of $J_{0}(K)$ of the form $\left\{v_{0}, v_{e}\right\},\left\{v_{e}, v_{1}\right\},\left\{v_{0}, v_{f}\right\},\left\{v_{f}, v_{1}\right\},\left\{v_{e}, v_{f}\right\}$, where $e=\left\{v_{0}, v_{1}\right\} \in J_{1}(K)$, $e \subset f, f \in J_{2}(K)$ and faces $J_{2}(S)$ the 3-element subsets of $J_{0}(S)$ of the form $\left\{v_{0}, v_{e}, v_{f}\right\},\left\{v_{1}, v_{e}, v_{f}\right\}$, where $e=\left\{v_{0}, v_{1}\right\} \in J_{1}(K), e \subset f, f \in J_{2}(K)$. (In a geometric realisation of $K$, the barycentric subdivision $S=S(K)=K^{\prime}$ is the 
simplicial complex whose 2-simplexes have vertices the barycentres of $J_{0}(K), J_{1}(K)$ and $J_{2}(K)$ ). We then have the action of the cartographic group on $\mathscr{F}(S)$ as $\mathscr{C}_{2}{ }^{S}$ and for $L \in \mathscr{F}(S)$ the generators $\varrho_{0}^{\prime}, \varrho_{1}^{\prime}, \varrho_{2}^{\prime}$ of $\mathscr{C}_{2}^{+, S}$ clearly satisfy the relations,

$$
\begin{aligned}
\left(\varrho_{0}^{\prime}\right)^{\nu_{0}(L)}(L) & =L, \\
\left(\varrho_{1}^{\prime}\right)^{2}(L) & =L, \\
\left(\varrho_{2}^{\prime}\right)^{3}(L) & =L .
\end{aligned}
$$

Above, we have written the set $J_{0}(S)$ as a union of three families: a vertex is of one of type $v \in J_{0}(K)$, type $v_{e}, e \in J_{1}(K)$ or type $v_{f}, f \in J_{2}(K)$. By identifying the elements of $J_{0}(S)$ of each type we obtain a simplex $T$ with for each $L \in \mathscr{F}(T)$, $\nu_{0}(L)=2, \nu_{2}(L)=3$. Indeed, $\mathscr{C}_{2}^{+, T}=S_{3}=\left\langle t_{0}, t_{1}, t_{2} \mid t_{0}^{2}=t_{1}^{2}=t_{2}^{3}=t_{2} t_{1} t_{0}=1\right\rangle$, the symmetric group on 3 elements. Hence, mapping $\mathscr{C}_{2}^{+, S}$ to $\mathscr{C}_{2}^{+, T}$ by the induced action, we have a surjection with kernel $\Gamma$, say:

$$
1 \rightarrow \Gamma \rightarrow \mathscr{C}_{2}^{+, S} \rightarrow S_{3} \rightarrow 1 \text {. }
$$

As $t_{0}^{2}=t_{1}^{2}=1$ we have both $\tilde{\varrho}_{0}=\left(\varrho_{0}^{\prime}\right)^{2}$ and $\tilde{\varrho}_{1}=\varrho_{1}^{\prime}\left(\varrho_{0}^{\prime}\right)^{2} \varrho_{1}^{\prime} \in \Gamma$. Moreover, a direct computation using $\left(\varrho_{1}^{\prime} \varrho_{0}^{\prime}\right)^{3}=1$ shows $\tilde{\varrho}_{0}$ and $\tilde{\varrho}_{1}$ to be invariant under conjugation by the elements of $\mathscr{C}_{2}^{+, S}$. Now, the quotient $\left(\mathscr{C}_{2}^{+, S} / \Gamma\right)=S_{3}$ has 6 elements, but then a direct computation shows that the quotient $\mathscr{C}_{2}^{+, S} /\left\langle\tilde{\varrho}_{0}, \tilde{\varrho}_{1}\right\rangle$ does also, so that $\Gamma=\left\langle\tilde{\varrho}_{0}, \tilde{\varrho}_{1}\right\rangle$. To every flag $L=(v, e, f) \in \mathscr{F}(K)$ is associated a unique flag $L^{\prime}=\left(v^{\prime}, e^{\prime}, f^{\prime}\right) \in \mathscr{F}(S)$ with $v^{\prime}=v, e^{\prime}=\left\{v, v_{e}\right\}$ and $f^{\prime}=\left\{v, v_{e}, v_{f}\right\}$. It is easy to see that the flag $\varrho_{0}(L)$ is then associated to $\varrho_{0}\left(L^{\prime}\right)$ and the flag $\varrho_{1}(L)$ to $\varrho_{1}\left(L^{\prime}\right)$. As $\varrho_{0}$ and $\varrho_{1}$ generate $\mathscr{C}_{2}^{+, K}$, these remarks show that there is a surjection of $\Gamma$ on $\mathscr{C}_{2}^{+, K}$. Notice that if $L^{\prime}=\left(v, e^{\prime}, f^{\prime}\right)$ with $v \in J_{0}(K)$ then $\nu_{0}\left(\varrho_{1}^{\prime}\left(L^{\prime}\right)\right)=4$ so that indeed $\left(\tilde{\varrho}_{1}\right)^{2}\left(L^{\prime}\right)=L^{\prime}$. In [BauItz], an example is given which shows that $\Gamma$ is not isomorphic to $\mathscr{C}_{2}^{+, K}$ in general.

Consider a cell complex $K$ and the set of edges $J_{1}^{\prime}(K)$ of $S=S(K)$ of the form $\left\{v_{0}, v_{e}\right\},\left\{v_{e}, v_{1}\right\}$, where $e=\left\{v_{0}, v_{1}\right\} \in J_{1}(K)$. Then $J_{1}^{\prime}(K)$ has $2 A$ elements, where $A$ is the cardinality of $J_{1}(K)$. Let $V_{\nu_{0}}$ be the number of vertices of valency $\nu_{0}$ and $F_{\nu_{2}}$ be the number of faces of valency $\nu_{2}$. Then

$$
\begin{aligned}
2 A & =\sum_{\nu_{0}} \nu_{0} V_{\nu_{0}}=\sum_{\nu_{2}} \nu_{2} F_{\nu_{2}}, \\
V & =\sum_{\nu_{0}} V_{\nu_{0}}, F=\sum_{\nu_{2}} F_{\nu_{2}},
\end{aligned}
$$

so that the number of odd valency vertices and faces is even. We remark with [ShVo] that the positively oriented flags (written "flags"," singular "flag ") of $K$ are in 1 to 1 correspondence with the oriented edges of $K$. An edge may be oriented in two ways. Hence the flags ${ }^{+}$are in 1 to 1 correspondence with the elements of $J_{1}^{\prime}(K)$. The total number of flags ${ }^{+}$is therefore $2 A$ and one may view the group $\mathscr{C}_{2}^{+, K}$ as a subgroup $\mathscr{G}=\mathscr{G}(\mathscr{K})$ of the symmetric group $S_{2 A}$ of order $2 A$, see [Baultz] for more details. As in [BauItz], we let $H$ be the group of symmetries of $K$ given by the centraliser of $\mathscr{G}$ in $S_{2 A}$, that is the set of permutations of the $2 A$ flags $^{+}$commuting $^{+}$ with the action of $\mathscr{G}$. Denote by $p, q, r$ the respective orders of the $\varrho_{i}, i=0,1,2$. Then $q=2$ and $p, r$ are respectively the smallest common multiples of the valencies 
of the vertices and faces. Following [ShVo], we say that $K$ is balanced if all the valencies of its vertices are equal, and hence equal to $p$, and if all the valencies of its faces are also equal, to $r$. Let $B$ be the stabiliser in $\mathscr{G}$ of any flag ${ }^{+}$(the action of $\mathscr{G}$ on the flags ${ }^{+}$is transitive). We say that $K$ is regular if $B$ is trivial. We have the following propositions whose proofs may be found in [BauItz],

Proposition 4. The order of the symmetry group of a cell complex divides twice the number of its edges and the complex is regular if and only if its symmetry group is isomorphic (but not identical) to its oriented cartographic group.

Proposition 5. Let $g=1-\chi / 2$, where $\chi$ is the Euler characteristic. Then for $g>1$ the order of the symmetry group of a regular cell complex is at most $84(g-1)$.

There is an intrinsic description of the symmetry group $H$ of $K$. Choose a flag $L \in \mathscr{F}=\mathscr{F}(K)$, let $B_{L} \subset \mathscr{G}$ be its isotropy group in $\mathscr{G}$ and consider the normaliser $\operatorname{Norm}\left(B_{L}\right) \subset \mathscr{G}$, that is the group of $g \in \mathscr{G}$ such that $g B_{L} g^{-1}=B_{L}$. Clearly $B_{L}$ is an invariant subgroup of $\operatorname{Norm}\left(B_{L}\right)$. We have,

Proposition 6. The symmetry group $H$ is isomorphic to $\operatorname{Norm}\left(B_{L}\right) / B_{L}$.

Let us now place this discussion in the context of dessins on compact oriented surfaces. As in [Gr, ShVo], a dessin $D$ on a compact oriented surface $\Sigma$ is a pair $D=(K(D),[\imath])$, where $K(D)$ is (the underlying polygon of) a connected 1-complex and $[\imath]$ is an isotopical class of inclusions $\imath: K(D) \hookrightarrow \Sigma$. One supposes as in [ShVo] that the complement of $\imath\left(K_{0}(D)\right)$ in $\imath(K(D))$ is a finite disjoint union of open segments, together with the regularity assumptions. We may then, as in Sect. 5, speak of vertices, edges and faces in the usual sense of a graph and it is clear that by considering $G=\imath(K(D))$ as giving a cellular decomposition of $\Sigma$ we obtain the oriented cartographic group $\mathscr{G}$ as a subgroup of $S_{2 A}, A=\operatorname{card} J_{1}(G)$.

By realising on $\Sigma$ the subdivision described in the present section, of $G$ into a 2-simplex $S=S(G)$, we obtain a triangulation of $\Sigma$ whose vertices are divided into three mutually exclusive families: a vertex is a $v \in J_{0}(G)$, a $v_{e}, e \in J_{1}(G)$ or a $v_{f}$, $f \in J_{2}(G)$. We have already seen in Sect. 1 how a clean Belyi function gives rise to a dessin. Here we are now ready to carry out the converse construction: the orientation of the surface gives rise to a bicolouration of the triangles. One constructs a function $\beta_{D}$ from $\Sigma$ to $\mathbb{P}_{1}(\mathbb{C})$ so as to send each vertex $v \in J_{0}(G)$ to $\infty$, each vertex $v_{e}$, $e \in J_{1}(G)$ to 1 and each vertex $v_{f}, f \in J_{2}(G)$ to 0 , with the triangles of one colour mapping onto $\mathfrak{h}$ and of the other colour onto $\mathfrak{h}^{-}$. This construction of Belyi [Be] is discussed in [ShVo] and in depth in [Wo1], where it is shown that a conformal structure on $\Sigma$ exists such that this function $\beta_{D}$ gives rise to a clean Belyi function defined over a number field on a smooth algebraic curve structure underlying $\Sigma$. We therefore recover the Grothendieck dessin $G$ by taking $\beta_{D}^{-1}[1, \infty]$ as in the discussion of Sect. 1.

We remark with [ShVo] that as $\mathscr{G}$ acts on the oriented edges of $G$ one may as in [ShVo, p. 207, 1.1.4] define the action of $\mathscr{G}$ on the positively oriented flags, with no regularity assumptions: so that $G$ may have loops and trees (see also our remarks below on abstract cell complexes).

The context of abstract cell complexes. In this subsection we remark that an analogous construction of the oriented cartographic group can be carried out using the language of abstract cell complexes as defined for example in [AhlSa, Ch. 1§7]. It is in this spirit that the oriented cartographic group is introduced in [BauItz]. A (finite, abstract) 
cell complex $K$ consists of two finite sets, the set $E=J_{1}(K)$ of edges and the (nonempty) set $F=J_{2}(K)$ of faces. Each element $e \in E$ has an inverse $e^{-1} \in E$ and each $f \in F$ also has an inverse $f^{-1} \in F$. One supposes that $\left(e^{-1}\right)^{-1}=e,\left(f^{-1}\right)^{-1}=f$, $e^{-1} \neq e$ and $f^{-1} \neq f$. To each face $f$ is assigned its boundary, given by a cyclically ordered set $e_{1} e_{2} \ldots e_{m}, m \geq 1$ of edges $e_{i}, i=1, \ldots, m$, where the inverse cycle $e_{m}^{-1} \ldots e_{2}^{-1} e_{1}^{-1}$ is assigned to $f^{-1}$. The vertices $V=J_{0}(K)$ of $K$ are of two types, inner and border vertices, and can be defined using the sets $E$ and $F$. Namely, we call a successor of the edge $e$ any edge appearing immediately after $e$ in a boundary of a face. A cyclically ordered set $v=\left(e_{1}, e_{2}, \ldots, e_{n}\right)$ is called an inner vertex if $e_{i}$ has $e_{i-1}^{-1}$ and $e_{i+1}^{-1}$ (indices taken modulo $n$ ) as successors. If the same condition holds for $1<i<n$, while $e_{1}$ and $e_{n}$ have only the successors $e_{2}^{-1}$ and $e_{n-1}^{-1}$ respectively, then $v$ regarded as a linearly ordered set is called a border vertex.

In the exposition of [AhlSa, Ch. 1§7] it is assumed that each edge of $K$ occurs either once or twice as an element of a boundary and that $K$ is connected. If an edge appears in two places, it therefore has two successors counted with multiplicity, i.e. they may be identical.

With these conventions, it is clear that every edge can be assigned a vertex (qualitatively, its terminal point) as follows: starting from an edge $e$ we find its neighbours in a vertex by taking the inverses of its successors. This rule can be iterated in both directions until the cycle closes, or until we hit sides with only one successor.

The faces of the cell complex are divided into pairs $f, f^{-1}$. A choice of notation whereby one out of each of these pairs is denoted by $f$ and the other by $f^{-1}$, constitutes an orientation of $K$ (see [AhlSa, p. 94, $\S 40]$ ). The orientation is called coherent if each edge $e$ appears at most once in the whole system of boundaries of the faces $f$ : so if $e$ appears twice in the whole system of boundaries, then it appears as the boundary of a face $f_{1}$ and of a face $f_{2}^{-1}$. A cell complex $K$ is called orientable if it has a coherent orientation. Now, supposing that $V$ contains no border vertices and that $K$ is orientable, we choose an orientation of $K$. That is, from each pair of faces, $f, f^{-1}, f \in F$, we retain just one and this in such a way that every edge $e$ appears once on the boundary of a face. Intuitively, the edges $e$ and $e^{-1}$ appear as paired sides of neighbouring faces. The vertices are now the cyclically ordered sets $v=\left(e_{1}, e_{2}, \ldots, e_{n}\right)$ such that $e_{2+1}^{-1}$ is a successor of $e_{i}$, that is $e_{i+1}^{-1}$ appears immediately after $e_{i}$ on the boundary of a retained face, with indices taken modulo $n$. This representation of $v$ is unique. To $e \in E$ is associated one vertex $v$ (its terminal point as described above) and $v$ has a unique representation as $v=\left(e_{1}, e_{2}, \ldots, e_{n}\right)$ with $e=e_{i}$ some $i=1, \ldots, n$. We let $\kappa_{0}$ be the map $e \mapsto e_{i+1}$, where the index is taken modulo $n$. We let $\kappa_{1}$ be the map which to the edge $e$ associates the edge $e^{-1}$ and define $\kappa_{2}$ by $\kappa_{2} \kappa_{1} \kappa_{0}=1$. Notice that these actions are defined without regularity assumptions. We have already remarked that the oriented edges of a cell complex are in one to one correspondence with its positively oriented flags. The edges of our abstract cell complex are therefore in one to one correspondence with the positively oriented flags. The action of the group generated by $\kappa_{0}, \kappa_{1}, \kappa_{2}$ corresponds to the action of the oriented cartographic group induced by this correspondence (see [ShVo, p. 207, 208], although notice that the illustrations in this reference indicate that to each edge was associated its initial, rather than its terminal, point). In this way, the cellular decomposition of a compact connected oriented surface is generated by the transitive action of an oriented cartographic group on a set of positively oriented flags. 
Notice that the initial discussion of this section is recovered from an oriented abstract cell complex by forgetting the distinction between initial and terminal point (which is not necessary to our definition of an abstract simplicial complex). To each edge $e$ we may associate a set of two vertices whose elements are the terminal point of $e$ and the terminal point of $e^{-1}$ : in this sense we say that $e$ contains two vertices and consider it as being this two point set. It is clear that $e$ and $e^{-1}$ now contain the same vertices and are no longer distinguished. We say that $e$ is an edge of the face $f$ if $e$ occurs in the boundary of $f$ (each edge will now occur in two boundaries) and that $v$ is vertex of $f$ if $v$ is contained in one of the edges of $f$. We consider the faces to be given by their vertex sets so that $f$ and $f^{-1}$ are no longer distinguished.

\section{Some Examples Coming from the Platonic Solids}

With the notation of Sect. 5, the inequalities $2 A \leq p V$ and $2 A \leq r F$ lead to $\chi \geq 2 A\left(\frac{1}{p}+\frac{1}{2}+\frac{1}{r}-1\right)$. Hence, if $\left(\frac{1}{p}+\frac{1}{2}+\frac{1}{r}\right)>1$ we are in genus 0 (that is the case of a spherical triangle group in the context of Sect. 1). The genus 0 balanced cases correspond to the Platonic solids and we have the table $(n \geq 2)$ :

\begin{tabular}{rrrrrrrr}
\hline$p$ & $q$ & $r$ & $V$ & $E$ & $F$ & $\mathscr{G}$ & $|\mathscr{G}|$ \\
\hline 2 & 2 & $n$ & $n$ & $n$ & 2 & $D_{n}$ & $2 n$ \\
$n$ & 2 & 2 & 2 & $n$ & $n$ & $D_{n}$ & $2 n$ \\
3 & 2 & 3 & 4 & 6 & 4 & $A_{4}$ & 12 \\
4 & 2 & 3 & 6 & 12 & 8 & $S_{4}$ & 24 \\
3 & 2 & 4 & 8 & 12 & 6 & $S_{4}$ & 24 \\
5 & 2 & 3 & 12 & 30 & 20 & $A_{5}$ & 60 \\
3 & 2 & 5 & 20 & 30 & 12 & $A_{5}$ & 60 \\
\hline
\end{tabular}

Consider the regular polyhedra on the Riemann sphere in Euclidean 3-space whose faces are equilateral triangles: namely, the regular tetrahedron $\left(\mathscr{G}=A_{4}\right)$, octahedron $\left(S_{4}\right)$ and icosahedron $\left(A_{5}\right)$. The Riemann sphere can be considered as the concentric circumscribed sphere whose centre is the centre of symmetry of the polyhedron. These polyhedra correspond to the Schläfli symbol of the form $\{3, \nu\}$ : i.e. we have a net of equilateral triangles $\nu$ surrounding each vertex. One has $\nu=3$ for the tetrahedron, $\nu=4$ for the octahedron and $\nu=5$ for the icosahedron.

Given any face, we can consider its barycentric subdivision, as an equilateral triangle $T$, into six smaller triangles with angles $\frac{\pi}{2}, \frac{\pi}{3}$ and $\frac{\pi}{6}$. These smaller triangles have three types of vertices: $v$ a vertex of $T, v_{e}$ a vertex in the middle of an edge of $T$ and $v_{f}$ a vertex in the middle of a face of $T$. For the six small triangles, the angle at the vertices of type $v$ is $\frac{\pi}{6}$, at type $v_{e}$ is $\frac{\pi}{2}$ and at type $v_{f}$ is $\frac{\pi}{3}$. We may project each of these triangles on the circumscribed sphere using the sphere centre as the centre of projection. One obtains in this way a spherical triangulation of the Riemann sphere formed by arcs of great circles. The angles of the small spherical triangles at the vertices of the polyhedron (on the sphere before projection) are given by $\frac{\pi}{\nu}$, 
where $\nu$ is (as above) the number of faces around a vertex. The other angles of the smaller triangles are preserved under projection so that we have angles $\frac{\pi}{2}, \frac{\pi}{3}, \frac{\pi}{\nu}$ for the small spherical triangles. Notice that the large spherical triangles obtained from the faces of the polyhedron have angles $\left(\frac{2 \pi}{\nu}, \frac{2 \pi}{\nu}, \frac{2 \pi}{\nu}\right)$. There is a bicolouration of the small spherical triangles and a natural Belyi function $\beta_{\nu}$ mapping every triangle of one colour to $\mathfrak{h}^{+}$while mapping every triangle of the other colour to $\mathfrak{h}^{-}$. This Belyi function is given by the inverse of a Schwarz triangle function and has ramification numbers 2,3 and $\nu$ over its respective ramification points.

It is possible to construct a compact connected Riemann surface $\Sigma_{\nu}$ which as a branched covering of the Riemann sphere has branch points of the same and minimal order exactly at the vertices of the polyhedra (one vertex of type $v$ per small spherical triangle). Composing the natural projection from this surface onto the sphere with the Belyi function $\beta_{\nu}$ one would obtain a Belyi function $\beta_{\nu}^{\Sigma}$ on the surface with ramification numbers 2,3 and $\nu \times S$, where $S$ is the number of sheets. Now, for the tetrahedron, there are 4 vertices with 3 faces at each vertex so accounting for an angle $\pi$ at this vertex. The surface $\Sigma_{3}$ must therefore have a branch point of number 2 at each vertex in order to fill up an angle of $2 \pi$. By the formula of Riemann-Hurwitz, the surface $\Sigma_{3}$ then has genus 1 . Indeed, by cutting along the edges meeting at a vertex, one may open out the tetrahedron as a triangle in Euclidean space subdivided into 4 smaller triangles. On doubling ( $\Sigma_{3}$ has 2 sheets) the triangle by reflecting it in one of its sides, we obtain 8 triangles. By identifying sides congruent under the reflection we obtain as expected a torus triangulated by 8 equilateral triangles. In a similar fashion, for the octahedron there are 6 vertices with 4 faces at each vertex filling up an angle of $\frac{4 \pi}{3}$, so that $\Sigma_{4}$ has a branch point of number 3 at each vertex. The genus of $\Sigma_{4}$ is therefore 4 and the triangulation induced from that of the polyhedron has 24 triangles. For the octahedron, there are 12 vertices with 5 faces at each vertex filling up an angle of $\frac{5 \pi}{3}$. The branch points therefore have number 6 and $\Sigma_{6}$ has genus 25 with an induced triangulation of 120 triangles.

By the discussion of Sect. 1, the ramification numbers of the Belyi function $\beta_{\nu}^{\Sigma}$ on $\Sigma_{\nu}$ enable one to deduce that $\Sigma_{\nu}$ is a quotient of a simply connected domain by a subgroup of finite index in a Fuchsian triangle group of signature $(2,3,2 e)$, where $2 e=\nu \times S$ turns out to be the number of edges of the polyhedron. This simply connected domain is the complex plane for the tetrahedron, the Poincaré upper half plane for the octahedron and icosahedron, whilst $2 e=6,12$ and 30 respectively for the tetrahedron, octahedron and icosahedron. The nature of the ramifications ensures that these are universal covering groups. Recall (see Sect. 4) that the Fermat curve $F_{e}$ is also a quotient of the Poincaré upper half plane by a subgroup of finite index in the Fuchsian triangle group of signature $(2,3,2 e)$.

In the case of the tetrahedron (only), $\Sigma_{3}$ with the equilateral conformal structure is in fact the Fermat curve [AuItz]. Indeed, consider the triangulation of $\Sigma_{3}$ that we obtained above as a pull-back of the equilateral triangular faces of the corresponding polyhedron. One may now endow $\Sigma_{3}$ with the conformal structure whereby this triangulation is itself equilateral. The Schwarz map

$$
t=\int_{0}^{x} \frac{d u}{u^{2 / 3}(1-u)^{5 / 6}}
$$


maps the half-plane $\operatorname{Im} x>0$ into a triangle with angles $\frac{\pi}{2}, \frac{\pi}{3}, \frac{\pi}{6}$, one of triangles of the barycentric subdivision of an equilateral triangle. In terms of the variable $x$, the holomorphic differential $d t$ is therefore given by $\omega=\frac{d x}{x^{2 / 3}(1-x)^{5 / 6}}$. Now, Klein
calculated the inverse

$$
x=x(y)=\frac{y^{3}\left(y^{3}+8\right)^{3}}{\left(y^{6}-20 y^{3}-8\right)^{2}}
$$

of the spherical triangle map for the angles $\frac{\pi}{2}, \frac{\pi}{3}, \frac{\pi}{3}$. Here the normalization is such that each vertex at the angle $\frac{\pi}{2}$ is mapped to $x=\infty$, at the angle $\frac{\pi}{3}$ to $x=1$ and at the angle $\frac{\pi}{6}$ to $x=0$. These spherical triangles are pulled back by $\beta_{3}$ to the triangles of the above barycentric subdivision of the equilateral triangulation. Hence to calculate the equation of $\Sigma_{3}$ we need to rewrite $\omega$ in terms of $y$. We obtain

$$
\left(6 \frac{d y}{d t}\right)^{2}=1-y^{3}
$$

which is the affine equation of the elliptic Fermat curve $F_{3}$. For the cases $\nu=4,5$ the same calculation has been commented in [Aultz]. We shall meet the idea behind the computation again in Sect. 7.

\section{Rational Triangular Billiards}

In this section, we explain how the rational triangular billiards considered in [AuItz] in the context of the pseudo-integrable systems of Richens and Berry [RiBe] provide natural examples of some of the considerations central to the preceding sections. We rely on the older exposition of Hobson [Hob] for our introductory remarks below. For a recent survey of problems linked to the trajectories of a point mass in a billiard, see $[$ Ber].

Consider the motion of a single point mass moving elastically in 2 dimensions and at constant speed (the absolute value of the velocity) inside a euclidean triangle $R$ with interior angles the rational multiples of $\pi: \frac{\pi p_{0}}{q_{0}}, \frac{\pi p_{1}}{q_{1}}, \frac{\pi p_{\infty}}{q_{\infty}},\left(p_{i}, q_{i}\right)=1, i=0,1, \infty$. In particular, we have $\frac{p_{0}}{q_{0}}+\frac{p_{1}}{q_{1}}+\frac{p_{\infty}}{q_{\infty}}=1$. With energy conserved, the motion is confined to the reduced phase space $X=R \times[0,2 \pi)$ of points $x=\left(q_{1}, q_{2}, \phi\right)$, where $x=\left(q_{1}, q_{2}\right) \in R$ is the position of the point mass and $\phi$ is the velocity direction. When the point mass meets a side of the triangle it undergoes a reflection so that its perpendicular velocity changes sign. We do not consider the singular trajectories hitting the corners.

Such a motion can be developed in a straight line as follows. From an initial point $x=\left(q_{1}, q_{2}\right) \in R$, draw a ray from $\left(q_{1}, q_{2}\right)$ in the direction $\phi$. Reflect $R$ across the side through which this ray passes. Repeat this operation to obtain a sequence of triangles along the ray. It is clear that we can study the possible motions by studying the properties of rays passing through these sequences as the initial point varies. The union of such triangles is contained in the configuration $A$ of possibly overlapping triangles obtained by reflecting $R$ across each of its sides and then iterating this operation for the images. 
As $R$ has the rational angles $\frac{\pi p_{i}}{q_{i}}, i=0,1, \infty$, it is straightforward to check that the elements of $A$ have $2 Q, q=$ l.c.m. $\left(q_{0}, q_{1}\right)$ possible orientations. We retain in $A$ only a system of representatives for these orientations. We may divide the array $A$ into the subarray $A_{+}$of polygons obtained from $R$ by an even number of reflections and the subarray $A_{-}$of polygons obtained from $R$ by an odd number of reflections. Write therefore $A=R_{1}^{+} \cup R_{1}^{-} \cup \ldots \cup R_{Q}^{+} \cup R_{Q}^{-}$.

Our remarks show that the velocity of the single point mass can take at most $2 Q$ values of equal length. The reflections $s_{1}, s_{2}$ in any two distinct sides of $R$ meeting at an angle $\frac{\pi p}{q},(p, q)=1$, satisfy

$$
s_{1}^{2}=s_{2}^{2}=\left(s_{1} s_{2}\right)^{q}=1
$$

(in the sense that $\left(s_{1} s_{2}\right)^{q}$ sends $R$ to a triangle of the same orientation lying above it in a cyclic neighbourhood of the fixed point vertex) and give a faithful representation of the dihedral group $D_{2 q}$, the group generated by the reflections in two lines making an angle $\frac{\pi}{q}$. On combining the actions of the $D_{2 q_{i}}, i=1,0, \infty$ we realise an action of the group $D_{2 Q}$ on the possible velocity directions. Now, $A$ forms a possibly overlapping array of $2 Q$ triangles with some free (unpaired) sides. Let $E_{i}^{ \pm}$be a free side of $R_{\imath}^{ \pm}$, $i=1, \ldots, Q$. If, in the configuration $A$, reflection of $R_{i}^{ \pm}$through $E_{i}^{ \pm}$gives a triangle with the same orientation as $R_{j}^{\mp}$, then reflection of $R_{j}^{\mp}$ through just one of its sides $E_{j}^{\mp}$ gives a triangle with the same orientation as $R_{\imath}^{ \pm}$. We pair, by labelling identically, the parallel sides $E_{j}^{ \pm}$and $E_{j}^{\mp}$. All the sides of the elements of $A$ are now identified in pairs (via the above action of $D_{2 Q}$ ) and one may form a triangulated compact oriented surface, associated to the space of an abstract polyhedron with faces comprising $2 Q$ copies of the euclidean triangle $R$, by fitting together $2 Q$ oriented curvilinear triangles according to this pairing [AuItz]. The triangles have in common $3 Q$ sides. Since for $i=0,1, \infty$, a vertex of angle $\frac{\pi p_{i}}{q_{2}},\left(p_{i}, q_{i}\right)=1$, gives rise to a fixed point of order $2 q_{\imath}$ for the above local action of $D_{2 q_{\imath}}$, there are $\sum_{i=0,1, \infty} \frac{Q}{q_{i}}$ distinct vertices in this triangulation. Notice that this is a purely combinatorial construction and that the same surface could have been modelled from unoverlapping copies of the triangle with angles $\frac{\pi}{q_{0}}, \frac{\pi}{q_{1}}, \frac{\pi}{q_{\infty}}$ (which are most often hyperbolic; see the end of the present section). The Euler characteristic $\chi$ and genus $g$ of this surface are given by

$$
\chi=2-2 g=\left(\sum_{i=0,1, \infty} \frac{Q}{q_{\imath}}\right)-Q .
$$

We have therefore a topological description of a surface and an orientation for it, together with the formula for its genus. As explained in [AuItz] the configuration $A$ contains branch data which codes for a specific birational equivalence class of curves: namely, writing $\frac{p_{\imath}}{q_{i}}$ as $\frac{P_{\imath}}{Q}, i=0,1, \infty$, that of the Riemann surface $\Sigma_{R}=\Sigma\left(P_{0}, P_{1}\right)$ with equation

$$
y^{Q}=x^{Q-P_{0}}(1-x)^{Q-P_{1}} .
$$


To understand this, write as above $A=S_{1} \cup S_{2} \cup \ldots \cup S_{Q}$, where $S_{i}=R_{i}^{+} \cup R_{\imath}^{-}$. For $i=1, \ldots, Q$, the interior of the pair of triangles $S_{i}$ is equivalent, up to translation, rotation and rescaling, to the image of the pair of open triangles $\mathfrak{h}^{+} \cup \mathfrak{h}^{-}$of $\mathbb{P}_{1}(\mathbb{C})$ by a chosen branch of the Schwarz triangle map

$$
\tau=\int_{0}^{x} \frac{d u}{u^{1-P_{0} / Q}(1-u)^{1-P_{1} / Q}}
$$

so that $\tau$ is a complex coordinate in $S_{\imath}$. The passages $S_{i} \mapsto S_{i+1}, i=1, \ldots, Q-1$, $S_{Q} \mapsto S_{1}$ correspond to changes of branch of the multivalued function $\tau$. Writing the holomorphic differential $d \tau$ in the complex plane as the holomorphic differential $\frac{d x}{y}$ on a Riemann surface gives the equation for $\Sigma_{R}$, which as expected is paved by $2 Q$ triangles.

Assume $Q>3$. The euclidean triangle $R$ is the image by a conformal mapping, which may be continued to give a mapping holomorphic on $\mathfrak{h}$ using Schwarz' reflection principle, of the hyperbolic triangle $T_{Q}$ with angles $\frac{\pi}{Q}, \frac{\pi}{Q}, \frac{\pi}{Q}$. Recall now our discussion of Fermat curves in Sect. 4 so that $F_{Q}$ is the Fermat curve $X^{Q}+Y^{Q}=1$. Then a direct calculation shows that $\Sigma\left(P_{0}, P_{1}\right)$ is the image of $F_{Q}$ under the mapping

$$
\kappa:(X, Y) \mapsto(x, y)=\left(X^{Q}, X^{Q-P_{0}} Y^{Q-P_{1}}\right) .
$$

It is clear that the Belyi function $\beta_{Q}=X^{Q}$ (see Sect. 4) on $F_{Q}$ induces the Belyi function $\beta_{R}=x$ on $\Sigma\left(P_{0}, P_{1}\right)$. Inspection of the equation for $\Sigma\left(P_{0}, P_{1}\right)$ confirms that $\beta_{R}$ is indeed a Belyi function. The triangulation $G_{R}$ on $\Sigma_{R}$ with vertices $\beta_{R}^{-1}\{0,1, \infty\}$ is the image under $\kappa$ of the covered symmetric triangulation $K=K_{Q}$ on $F_{Q}$ by $2 Q^{2}$ triangles introduced in Sect. 4 and has the $2 Q$ triangles comprising $A$.

In Sect. 4 we saw that $\triangle=\triangle_{Q}$ acts on the triangulation of $F_{Q}$ as $[\triangle, \triangle]$ is normal in $\triangle$ [Wo1, §5.3]. Moreover, on passing to an extension $\bar{\Delta}$ of $\triangle$ of signature $(2,3,2 Q)$, we recover a representation of the action of the oriented cartographic group $\mathscr{C}_{2}^{+, S}$ where, as in Sect. $5, S=S(K)=K^{\prime}$ is the barycentric subdivision of the triangulation $K$ of $F_{Q}$ [Wo1, $\S 6$, ShVo]. The generator $\varrho_{0}^{\prime}$ of $\mathscr{C}_{2}^{+, S}$ satisfies $\left(\varrho_{0}^{\prime}\right)^{2 Q}=1$ so that $\tilde{\varrho}_{0}=\left(\varrho_{0}^{\prime}\right)^{2}$ has order $Q$ and descends to the action of the generator $\varrho_{0}$ of $\mathscr{G}_{R}=\mathscr{C}_{2}^{+, G_{R}}$. The generator $\varrho_{0}$ corresponds to the local action induced by the rotations $D_{2 q_{i}}^{+}$of $D_{2 q_{i}}$ around the vertices of angle $\frac{\pi p_{i}}{q_{i}}, i=0,1, \infty$, referred to above, and to a global action of $D_{2 Q}^{+}$.

We now explain how the construction in [AuItz] of a basis of holomorphic differentials on $\Sigma_{R}$ is closely related to the modular embedding construction of Sect. 2 of the present article. Recall that the triangle group $\triangle=\triangle(p, q, r)$ of signature $(p, q, r),\left(\frac{1}{p}+\frac{1}{q}+\frac{1}{r}\right)<1$ is the monodromy group of the hypergeometric differential equation of Gauss which has as a basis of its solution space two multivalent functions of the form:

$$
\int_{\gamma} \omega^{(1)}
$$


with

$$
\omega^{(1)}=\omega^{(1)}(x)=u^{-\mu_{0}}(u-1)^{-\mu_{1}}(u-x)^{-\mu_{2}} d u,
$$

where $\gamma$ is a certain integration path (one can take for example the two Pochhammer cycles around 1,0 and $0, x)$, the variable $x$ varies over $\mathbb{P}_{1}(\mathbb{C})-\{0,1, \infty\}$, and $\mu_{0}=\frac{1}{2}\left(1-\frac{1}{p}+\frac{1}{q}+\frac{1}{r}\right), \mu_{1}=\frac{1}{2}\left(1+\frac{1}{p}+\frac{1}{q}-\frac{1}{r}\right), \mu_{2}=\frac{1}{2}\left(1-\frac{1}{p}-\frac{1}{q}-\frac{1}{r}\right)$. Let $l$ be the least common multiple of the denominators of $\mu_{0}, \mu_{1}, \mu_{2}$ and let $\mu_{3}=2-\left(\mu_{0}+\mu_{1}+\mu_{2}\right)$. To construct the modular embedding one singles out those integers (that we call "admissible") $\nu \in(\mathbb{Z} / l \mathbb{Z})^{*}$ for which,

$$
r_{\nu}=:-1+\left\{\nu \mu_{0}\right\}+\left\{\nu \mu_{1}\right\}+\left\{\nu \mu_{2}\right\}+\left\{\nu \mu_{3}\right\}=1 \text {, }
$$

where $0 \leq\{a\}<1, a \in \mathbb{Q}$, denotes the fractional part of $a$. For each of the $2 t$ such integers $\nu$ we replace $\mu_{\imath}$ by $\left\{\nu \mu_{\imath}\right\}, i=0,1,2,3$ in the above integrand which becomes the differential of the first kind

$$
\omega^{(\nu)}=\omega^{(\nu)}(x)=u^{-\left\{\nu \mu_{0}\right\}}(u-1)^{-\left\{\nu \mu_{1}\right\}}(u-x)^{-\left\{\nu \mu_{2}\right\}} d u
$$

on a factor of the Jacobian of the curve $X(x)$ with affine equation,

$$
w^{l}=u^{l \mu_{0}}(u-1)^{l \mu_{1}}(u-x)^{l \mu_{2}} .
$$

This gives rise to the solution space of the Gauss hypergeometric differential equation associated to the triangle $T^{\nu}$ with angles the multiples of $\pi$ :

$$
\left|\left\{\nu \mu_{0}\right\}+\left\{\nu \mu_{2}\right\}-1\right|, \quad\left|\left\{\nu \mu_{1}\right\}+\left\{\nu \mu_{2}\right\}-1\right|, \quad\left|\left\{\nu \mu_{2}\right\}+\left\{\nu \mu_{3}\right\}-1\right|
$$

at the vertices corresponding to $x=0,1, \infty$ respectively. The triangles $T^{\nu}$ and $T^{-\nu}$ are isomorphic, so one has to make a choice modulo \pm 1 of the admissible $\nu$. The map of $T=T^{l}$ onto $T^{\nu}$, continued analytically to all of $\mathfrak{h}$, gives the $\nu^{\text {th }}$ component of the modular embedding [CoWo1].

In the construction of a basis of holomorphic differentials on $\Sigma_{R}$, with $R$ of angles the multiples $\frac{p_{0}}{q_{0}}, \frac{p_{1}}{q_{1}}, \frac{p_{\infty}}{q_{\infty}}$ of $\pi$, one singles out in [AuItz] those integers (also called "admissible") $\nu \in(\mathbb{Z} / Q \mathbb{Z})$ preserving the euclidean geometry; that is

$$
\left(\left\{\frac{\nu p_{0}}{q_{0}}\right\}+\left\{\frac{\nu p_{1}}{q_{1}}\right\}+\left\{\frac{\nu p_{\infty}}{q_{\infty}}\right\}\right)=1
$$

To each such integer $\nu$ one associates the differential of the first kind on $\Sigma_{R}$,

$$
\omega_{R}^{(\nu)}=u^{-1+\left\{\frac{\nu P_{0}}{Q}\right\}}(1-u)^{-1+\left\{\frac{\nu P_{1}}{Q}\right\}} d u
$$

and the euclidean triangle $R^{\nu}$ with angles

$$
\left\{\frac{\nu p_{0}}{q_{0}}\right\}, \quad\left\{\frac{\nu p_{1}}{q_{1}}\right\}, \quad\left\{\frac{\nu p_{\infty}}{q_{\infty}}\right\} .
$$

Indeed, the triangle map for $R^{\nu}$ is obtained by replacing $\omega_{R}^{(1)}$ by $\omega_{R}^{(\nu)}$ in the integral expression for $\tau$. Restricting to $\nu \in(\mathbb{Z} / Q \mathbb{Z})^{*}$ yields a basis on a factor of $\operatorname{Jac}\left(\Sigma_{R}\right)$.

Notice that the modular embedding construction in the hyperbolic case, when specialised to the fixed points of the corresponding triangle group, gives such a euclidean construction. Consider for example the point $x=0$ and suppose $\left(\mu_{0}+\mu_{2}\right)<1$ in the above construction. The admissible $\nu \in(\mathbb{Z} / l \mathbb{Z})^{*}$ are those 
for which $\sum_{j=0}^{3}\left\{\nu \mu_{i}\right\}=2$. At $x=0$, one has the new parameters $\mu_{0}^{\prime}=\mu_{0}+\mu_{2}$, $\mu_{1}^{\prime}=\mu_{1}, \mu_{2}^{\prime}=0, \mu_{3}^{\prime}=\mu_{3}$ corresponding to a euclidean triangle $R^{\prime}$ with angles $\frac{p_{0}}{q_{0}}=1-\left(\mu_{0}+\mu_{2}\right), \frac{p_{1}}{q_{1}}=1-\mu_{1}, \frac{p_{\infty}}{q_{\infty}}=1-\mu_{3}$, and the admissibility condition becomes $\left(\left\{\frac{\nu p_{0}}{q_{0}}\right\}+\left\{\frac{\nu p_{1}}{q_{1}}\right\}+\left\{\frac{\nu p_{\infty}}{q_{\infty}}\right\}\right)=1,(\nu, l)=1$, with $\omega^{\nu}(0)=\omega_{R^{\prime}}^{\nu}$.

Whereas in the hyperbolic case one associates to $T$ (see Sect. 2) a family of abelian varieties, one for each point of $T$ (and, by analytic continuation, of $\mathfrak{h}$ ), in the euclidean case there is only one such abelian variety associated to $R$. This degeneracy phenomenon is easily understood in terms of the holomorphic differentials associated to $T$ and $R$. In the hyperbolic case, the inequality $\left(\frac{1}{p}+\frac{1}{q}+\frac{1}{r}\right)<1$ ensures that $\mu_{2}$ is never zero, and the same applies to the $t$ differentials of the first kind obtained by replacing the $\mu_{i}, i=0,1,2,3$ by $\left\{\nu \mu_{i}\right\}, i=0,1,2,3$, where $\nu$ is admissible. Hence, for each $x$ these $t$ differentials give rise to differentials of the first kind on $\operatorname{Jac}(X(x))$. Although these $t$ differentials do not yield a full basis, they are sufficient to determine it. For more details see [CoWo1, Wo3]. By contrast, in the euclidean case, the admissibility condition gives rise to differentials of the first kind of the same form as those in the hyperbolic case, but with $\mu_{2}=0$, so that the dependency on $x$ in the integrand disappears and the differentials lie only on the Jacobian of $\Sigma_{R}$.

However, one can still associate to the rational billiard a family of abelian varieties as in the hyperbolic case. One applies the construction of Proposition 1, Sect. 1 of the present paper to the pair $(\Sigma, \beta)=\left(\Sigma_{R}, \beta_{R}\right)$, where $R$ is, as above, the euclidean triangle with angles $\pi \frac{p_{0}}{q_{0}}=\pi \frac{P_{0}}{Q}, \pi \frac{p_{1}}{q_{1}}=\pi \frac{P_{1}}{Q}, \pi \frac{p_{\infty}}{q_{\infty}}=\pi \frac{P_{\infty}}{Q}$. The Belyi function $\beta$ has ramification points $x=y=0$ of order $q_{0}$ over $\beta=0 ; x=1, y=0$ of order $q_{1}$ over $\beta=1$; and $x=\infty, y=\infty$ of order $q_{\infty}$ over $\beta=\infty$. Therefore, $\Sigma$ is the quotient of $\mathfrak{h}$ by a subgroup of finite index in a Fuchsian triangle group of signature $\left(q_{0}, q_{1}, q_{\infty}\right)$. The integer $t$ of Theorem 1, Sect. 2 is in this case the number of admissible $\nu \in(\mathbb{Z} / Q \mathbb{Z})^{*}$ for this signature, as defined in the above discussion of the present section. By this same theorem, the vertices $\beta^{-1}\{0,1, \infty\}$ of the triangulation are sent by the morphism $\psi: \Sigma \mapsto V$, where $V$ is a Shimura variety of dimension $t$, onto points of complex multiplication by a subfield of the cyclotomic field $\mathbb{Q}\left(\exp \left(\frac{\pi i}{Q}\right)\right)$.

As explained in Sect. 4 , the curve $\Sigma$ may also be represented as a quotient of $\mathfrak{h}$ by a subgroup of finite index in the Fuchsian triangle group with signature $(Q, Q, Q)$. Once again the construction of Proposition 1, Sect. 1 and Theorem 1, Sect. 2 can be applied, this time for $p=q=r=Q$. There are now $t_{Q}$, say, admissible elements of $(\mathbb{Z} / Q \mathbb{Z})^{*}$.

Notice that the integers $t$ and $t_{Q}$ are not necessarily equal. For example, when we make, for $Q=18$, the choice $\left(P_{0}, P_{1}, P_{\infty}\right)=(9,8,1)$, the triangle group of signature $(Q, Q, Q)=(18,18,18)$ is non-arithmetic and $t_{Q}=2$, whereas the triangle group of signature $\left(q_{0}, q_{1}, q_{\infty}\right)=(2,9,18)$ is arithmetic, so that $t=1$.

Acknowledgment. The first and the third author benefitted from the financial support of PROCOPE and the hospitality of the MSRI Berkeley. The third author wishes to thank the Centre d'études de Saclay for its support. 


\section{References}

[AhlSa] Ahlfors, L.V., Sario, L.: Riemann Surfaces. Princeton, New Jersey: Princeton Univ. Press, 1960

[AuItz] Aurell, E., Itzykson, C.: Rational billiards and algebraic curves. JGP 5, n. 2, 191-208 (1988)

[BauItz] Bauer, M., Itzykson, C.: Triangulations. Coll. en hommage à P. Cartier, 54ème Renc. Strasbourg, 1992

[Be] Belyi, G.: On Galois extensions of a maximal cyclotomic field. Math. USSR Izv. 14, No. 2, 247-256 (1980)

[Beh] Behr, H.: Über die endliche Definierbarkeit von Gruppen, J.f.d. reine u. angew. Math. 211, 116-122 (1962)

[Ber] Berger, M.: La mathématique du billard. Pour La Science, N. 163, 76-85 Mai, 1991

[CoWo1] Cohen, P., Wolfart, J.: Modular Embeddings for some non-arithmetic Fuchsian groups. Acta Arithmetica 56, 93-110

[CoWo2] Beazley Cohen, P., Wolfart, J.: Dessins de Grothendieck et variétés de Shimura, C.R. Acad. Sci. Paris, t. 315, Série I, 1025-1028 (1992)

[Cn] Cohn, H.: Conformal Mapping on Riemann Surfaces. London: Dover Pub., 1967

[De] Deligne, P.: Le groupe fondamental de la droite projective moins trois points. In: Galois Groups over $\mathbb{Q}$, e.d. Y. Ihara et al., MSRI Publ. 16, Berlin-Heidelberg-New York: Springer, 1989, pp. 79-297

[Dic] Encyc. Dic. of Math., Math. Soc. Japan, Cambridge, Mass. and London, Eng.: The MIT Press, 1980

[Ge] Gerstenhaber, M.: On the algebraic structure of discontinuous groups. Proc. Am. Math. Soc. 4, 745-750 (1953)

[Gr] Grothendieck, A.: Esquisse d'un programme. 1984, unpublished

[Hob] Hobson, A.: Ergodic properties of a particle moving inside a polygon. J. Math. Phys. 16, No. 11, (Nov. 1975)

[LuWe] Lundell, A.T., Weingram, S.: The Topology of CW Complexes. van Nostrand Reinhold, 1969

[MKS] Magnus, W., Karrass, A., Solitar, D.: Combinatorial Group Theory. London: Dover 1976

[P] Poincaré, H.: Théorie des groupes Fuchsiens. Acta Math. 1, 1-62 (1882)

[RiBe] Richens, P.J., Berry, M.V.: Pseudo-integrable systems in classical and quantum mechanics. Physica 2D, 495-512 (1981)

[RoSa] Rourke, C.P., Sanderson, B.J.: Introduction to Piecewise-Linear Topology. Ergeb. der Math., Band 69, Berlin, Heidelberg, New York: Springer 1972

[ShVo] Shabat, G.B., Voevodsky, V.A.: Drawing curves over number fields. In: The Grothendieck Festschrift, Vol. III, ed. P. Cartier et al., Progress in Math. 88, Basel, Boston: Birkhäuser, 1990, pp. 199-227

[Shi] Shimura, G.: Construction of class fields and zeta functions of algebraic curves. Ann. Math. 85, 58-159 (1967)

[VoSh] Voevodsky, V.A., Shabat, G.B.: Equilateral triangulations of Riemann surfaces, and curves over algebraic number fields. Soviet Math. Dokl. 39, No. 1, 38-41 (1989)

[Ta] Takeuchi, K.: Arithmetic triangle groups. J. Math. Soc. Japan 29, 91-106 (1977)

[Vi] Vignéras, M-F.: Arithmétique des Algèbres de Quaternions. LN in Math. 800, Berlin, Heidelberg, New York: Springer 1980

[We] Weil, A.: The field of definition of a variety. Am. J. Math. 78, 509-524 (1956)

[Wo1] Wolfart, J.: Mirror-invariant triangulations of Riemann surfaces, triangle groups and Grothendieck dessins: Variations on a thema of Belyi. Preprint of the Dept. Math. Joh. Wolf. Goethe-Univers., Frank./Main

[Wo2] Wolfart, J.: Eine arithmetische Eigenschaft automorpher Formen zu gewissen nichtarithmetischen Gruppen. Math. Ann. 262, 1-21, (1983)

[Wo3] Wolfart, J.: Werte hypergeometrische Funktionen. Invent. Math. 92, 187-216 (1988)

[Wo4] Wolfart, J.: Diskrete Deformationen Fuchsscher Gruppen und ihrer automorphen Formen. J.f.d. reine u. angew. Math. 348, 203- 220 (1986) 
